\title{
76TH ANNUAL SASKATCHEWAN CHRISTMAS BIRD COUNT - 2017
}

\author{
Alan R. Smith and Randi Edmonds \\ Box 154, Avonlea, SK SOH OCO \\ alanrandi@sasktel.net
}

We would like to dedicate the 2017 Christmas Bird Count to the memories of William Anaka and Lloyd Wilson pioneers in the study of birds in the Good Spirit Lake area.

\section{The Counts}

The number of counts declined by six counts over last year's 95. Total effort as measured by hours in the field and at feeders, or kilometres walked and driven was, however, about average for the last 20 years.

\section{The Weather}

Average minimum and maximum temperatures for the count period (with 2016-17 records in brackets) were -18 to $-12 \mathrm{C}(-17$ to $-11 \mathrm{C})$, wind speeds 9 to $18 \mathrm{~km} / \mathrm{h}$ ( 8 to 18 $\mathrm{km} / \mathrm{h}$ ), and snow depths 6 to $14 \mathrm{~cm}$ ( 6 to $14 \mathrm{~cm}$ ). As can be seen, weather conditions over the last two winters were nearly identical.

\section{The Birds}

The 127,798 birds counted was only slightly higher than 2016's 119,750, and about average for this century. The average number of species per count at 18.7 was also on par since 2000.

For the second year in a row, Gardiner Dam won the crown for the most species with 43; the runner up was Saskatoon with 42 species (plus two during the count period).

\section{Population Trends}

Waterfowl were up slightly from the previous year's lows. For example, Canada Geese rose from 17,219 to 22,801, Mallards 4,629 to 8,283.
Record high counts were set for Snow Goose and Greater Scaup.

Most raptors were recorded in their usual small numbers, but without the crops left in the fields as was the case in 2016-17, Northern Harriers dropped from 11 to none, and Short-eared Owls from 39 to 3. Snowy Owls were, however, much in evidence with 181 in 33 localities compared to 59 in 27 the previous winter. Regina tied the all-time record with 45 birds, while nearby Rouleau fell one short with 44.

The range expansion for two introduced species, Eurasian CollaredDove and House Finch, seems to have stalled as the only new CBC localities for these species should be regarded as "infilling" rather than expansion. The new site for the collared-dove was Floral; those for the finch were Dundurn and Grenfell. Notable was a new high count of 196 collared-doves from Swift Current.

Bohemian Waxwing dropped from 12,590 in 33 localities to 7,469 in 28 localities this past winter. This is reflected in the wild food crop which was reported "fair" or better in most areas in 2016, while fair or often poor in 2017.

Numbers of Common Redpoll were way up - 14,301 birds on 78 counts compared to 1,731 on 61 in 2016. Numbers of most other finches were, however, down.

\section{Rarities}

Rarities of note include our seventh and eighth Red-bellied Woodpeckers. One was on the Estuary North count and the other during the count period at Saskatoon. Our fourth Hermit Thrush was seen at Swift Current. No new species were recorded for the CBC - the all-time total remains at 191 species.
Note: for purposes of Saskatchewan Counts, as published herein, the count period extends from December 14 to January 5; Audubon counts include only those species recorded three days prior to and after the count day.

\section{Count areas and participants}

(names of compilers are in italics)

1. ARCHERWILL. Jeff Carlson, Monica Carlson, Shynese Carlson, Gerald Hiron, Susan Hiron, Audrey Hnetka, Perry Hnetka, Elaine Hughes, Dorothy Klettberg, Willie Klettberg, Annette Kozak, Cecil Reimer, Mary Reimer, Judy Revoy.

2. AVONLEA. Randi Edmonds, Don Robertson, Alan Smith, Blaine Sudom.

3. BALGONIE. Jared Clarke, Rowan Clarke, Teal Clarke, Ken Feltin, Tamara Harder, Sarah Ludlow, Kristen Martin, Brett Quiring, Jacqueline Roy, Wendy Woodard.

4. BIGGAR. Dale Booth, Mary-Jo Danychuk, Sheila Jezowski, Murray Newton, Mark Pickett, Nick Saunders, Lorrie Sielski, Lorne Sutherland, Phil Taylor, Guy Wapple, Rob Wapple.

5. BIRCH HILLS. Margaret Mareschal, Maurice Mareschal.

6. BORDEN-RADISSSON. Greg Fenty, Kyron Giroux, Ron Jensen, Elise Meister, Fred Meister.

7. BROADVIEW. Doug Boivin, Mary Ann Pearson, Barb Weidl, Don Weidl.

8. CATER: Beverly Beland, Orval Beland.

9. CHATSWORTH S.D. Herb Cross, Jean Cross, Charles Dyck, Donna Dyck, Robert Mess, Wionna Mess, George Murray, Laurie Murray, Marcel Pelletier, Kerri Rooke, Stewart Rooke, Tony Tangedal. 
10. CHRISTOPHER LAKE. Jeannie Walker.

11. CHURCHBRIDGE B. Dennis Petracek.

12. CLARK'S CROSSING. Christine

Christensen, Louise Cook, Lorne

Duczek, Andrew Elgin, Shelly Fisher, Bob Godwin, Dawn Hall, Marilyn Haskins, Jeff Jensen, Ron Jensen, Robert Johanson, Gwen Klypak, Heney Klypak, Brian McGill, Eileen McGill, Diane Murphy, John Patterson, Sylvia Raginski, Nick Saunders, Marten Stoffel, Geoff Urwin, Guy Wapple, Diane Wells, Michael Williams.

\section{CORONACH. Ryan Dudragne.}

14. CRAVEN. Hiroyuki Aoki, Dave Cameron, Joel Cherry, Blanche Cooper, Jim Cummings, Stephen Davis, Gail Fennell, Chris Harris, Louise Holloway, Phil Holloway, Kerri Irving, Jones Irving, Fran Kerbs, Laurie Koepke, Norbert Kossmann, Jackie Lane, Laurette Lane, Bob Luterbach, Barbara Mader, Kim Mann, Val Mann, Amelia McDonald, Jaret McDonald, Annie McLeod, Barry Mitschke, Ava Mund, Shasta Mund, Soren Mund, Curtis Pollock, Brett Quiring, Vera Raschke, Ed Rodger, Susan Rollins, Jacqueline Roy, Dan Sawatzky, Jeanette Taylor, Rob Taylor, Lorna Tyler, Mary Worel, Chris Whitall.

15. CREIGHTON. John Corden, Karen Prokopetz, Brenda Schmidt, Harvey Schmidt, Terry Smith.

16. CROOKED LAKE. Boyd Metzler.

17. CROOKED RIVER. Margaret Mehler, Morley Mehler.

18. CYPRESS HILLS PROVINCIAL PARK (Centre Block). David Larson, Margarette Larson, Melody Nagel-Hisey. 19. DENHOLM. Beverly Beland, Gerard Beland, Linda Beland, Orval Beland, Janice Mazurek.

20. DORINTOSH. Vicky Pryor, Joe Twidale, Lorraine Twidale, Tim Wahl.

21. DUNDURN. Ryan Dudragne, Ron Jensen, Nick Saunders, Phil Taylor.

22. DUVAL. Alan Martin, Lloyd Saul.
23. EASTEND. Loraine Armstrong, Roxie Binkley, Duane Bristow, Betty Davis, Tom Donald, Cynthia Fehr, Robert Gebhardt, June Higgins, Joan Hodgson, Harvey Johnson, Carolyn Templeton, Mary Thomson, Heidi Topham, Eldon Wig, Jack Wilkinson.

\section{EBENEZER B. Harvey Wegner.}

\section{ENDEAVOUR. Ed Harris.}

26. ESTEVAN. Barry Dies, Marilyn Dies, Craig Palmer, Larry Preddy, Reid Walton, Rose Walton, Guy Wapple, Sandra Wapple.

27. ESTUARY NORTH. Barry Cocks, Cathy Cocks, Dean Francis.

28. FENTON. Carman Dodge.

29. FLORAL. Mo Koeting, Guy Wapple.

30. FORT QU'APPELLE. Jean Ashcroft, Peter Ashcroft, Linda Banman, Jared Clarke, Teal Clarke, Rowan Clarke, Mark Geremia, Alice Isfan, Jerry Lasko, Jack Lowe, Marg Lowe, Kristen Martin, Jean McKenna, Alan Mlazgar, Wendy Paquin, Florence Pearpoint, Keith Stephens, Kim Stephens, Ted Stevenson, Colette Stushnoff, Marcel Vindevoghel.

31. GARDINER DAM. Ryan Dudragne, Krista Ellingson, Greg Fenty, Maxine Forsberg, Ron Jensen, Annie McLeod, Scott Olshanoski, Nick Saunders, Stan Shadick, Marten Stoffel, Phil Taylor, Guy Wapple.

32. GOOD SPIRIT LAKE. Joyce Anaka (non-participating compiler), Dorothy Riesz, Ray Riesz.

33. GRASSLANDS NATIONAL PARK. Kevin Moore.

34. GRAYSON. Charles Helm, Linda Helm, Jeanette Zimmer, Karl Zimmer.

35. GREENWATER. Bill Gudjonson, Brian Shuya, Helen Toovey.

36. GRENFELL. Ethel Reiger, Barb Weidl, Don Weidl.

37. HARRIS. Ryan Dudragne, Ron Jensen, Nick Saunders, Guy Wapple.

38. HAZLET. Ryan Dudragne.
39. HUDSON BAY. Judy Block, John Daisley, Agnes Lewellin, Bev Soules, John Zolkavich.

40. INDIAN HEAD. Kay Dixon, J. Dixon, Irvin Escott, David Gehl, Roberta Gehl, Sharon Hearn, Jim Jinks, Linda Jinks, Dan Loran, K. McClintock, Dora Nichols, Jim Rudack, Ruth Rudack, Lorette Schaeffer, Raymond Schaeffer, E. Schuurmans, Fred Skinner, C. Skinner, Brian Scott, Glenn Scott, Lorne Scott.

41. KENASTON. Doug Beckie, P. Lawrence Beckie, Tyler Beckie.

42. KENOSEE LAKE. Boyd Metzler, John Pollock.

43. KETCHEN NORTH. Dallas Fairburn.

44. KINDERSLEY NORTH. Jean Harris, Keith Harris.

45. KINLOCH. Andy Arnold, Caroline Arnold, Don Forbes, Carter Haroldson, Robert Haroldson, Clifford Logan, Doreen Wickstrom.

46. KYLE. Greg Nelson, Yvonne Nelson, Marten Stoffel, Dan Zazelenchuk.

47. LA RONGE. Kelly Gale, Michelle Gale, Mick Lessard, Linda Mikolenko, Ellen Paquin, Sid Robinson, John Schisler, Jan Shewchuk, Dave Smallwood.

48. LEADER NORTH. Daisy Meyers.

49. LEROY. Merv Hey, Alan Martin, Lloyd Saul.

50. LOVE - TORCH RIVER. Theresa Belliveau, Hal Birkett, Joan Birkett, Bert Dalziel, Joan Dalziel, Sara Dalziel, Scott Edwards, Andrea Fisher, Harold Fisher, Shelly Fisher, Taren Fisher, Elaine Inskster, Francis Inskster, Roseanne Kirkpatrick, Ray Kreutzwieser, Susan Kreutzwieser, Adrik Kurbis, Coulter Kurbis, Duane Kurbis, Renee Kurbis, Eilene L'Heureux, Lynn Matthews, Lillian Nesset, Eric Schrader, Janice Tourand, Leonard Turtle.

51. LUSELAND. Estelle Finley, Graeme Finley, Kim Finley, Liam Finley, Valerie Finley, Brent Honeker.

52. MEADOW LAKE. Bob Wilson, Ian Wilson. 
53. MELFORT. Bert Dalziel, Joan

Dalziel, Gordon Dodds, Shirley Dodds, Susan Dodds, Graydon Eskowich, Kim Eskowich, Wendy Eskowich, Travis Liddle.

54. MOOSE JAW. Chris Harris, Jeff Mander, Dan Sawatzky, Nick Selinger.

55. MOOSE MOUNTAIN. Arlie Altwasser, Darlene Altwasser, Bill Fletcher, Dorothy Fletcher, Doyle Thomas, Tanis Thomas, Val Thomas.

56. MORSE. Elizabeth Enns, Noel Enns, Stella Enns, Mike Francis, Roxanne Hagley, Randy McCulloch, Joel Priebe, Ken Priebe, Myrna Priebe, Lori Wilson.
57. NIPAWIN. Carol Blenkin, Nancy Budd, Vi Budd, Joyce Christiansen, Bert Dalziel, Joan Dalziel, Rick Douslin, Patti Gaertner, Jennette LeCuyer, George Lidster, Jeri McCleary, Peter McCleary, Doug Phillips, Shirley Phillips, Dorothy Reed, Fred Reed.

\section{NISBET FOREST, NORTHWEST.} Sandra Jewell.

59. NISBET FOREST, WEST. Kim Clark, Shamara Clark, Suzanne Clark.

60. ODESSA. Arden Curts, Denise Curts, Denny Curts.

61. PIKE LAKE. Donna Bruce, Dave Cook, Louise Cook, Lorne Duczek, Bob Girvan, Bob Godwin, Dawn Hall, Greg Hutchings, Audrey MacKenzie, Bill MacKenzie, Valerie Martz, Murray Morgan, John Patterson, Keith Paul,
Ljubica Stuglin, Stephan Stuglin, Nick Saunders, Phil Taylor, Guy Wapple, Michael Williams.

\section{PONTEIX. Ryan Dudragne.}

63. PRINCE ALBERT. Jim Bahr, Doug Braaten, Marie Braaten, Kim Clark, Shamara Clark, Bert Dalziel, Joan Dalziel, Carman Dodge, Andrea Fisher, Harold Fisher, Taren Fisher, Ham Greenwood, Don Guedo, Jeff Jensen, Ron Jensen, Gwen Klebek, Collin McGuire, Sonnet McGuire, John Rye, Vicki St. Germain, Warren St. Germaine, Don Weidl.

64. QU'APPELLE. Jean Ashcroft, Peter Ashcroft, Melanie Beattie, Cory Bennett, Christine Blair, Lillian Longpre, Paul Paulhus, Colette Stushnoff, Richard Stushnoff.

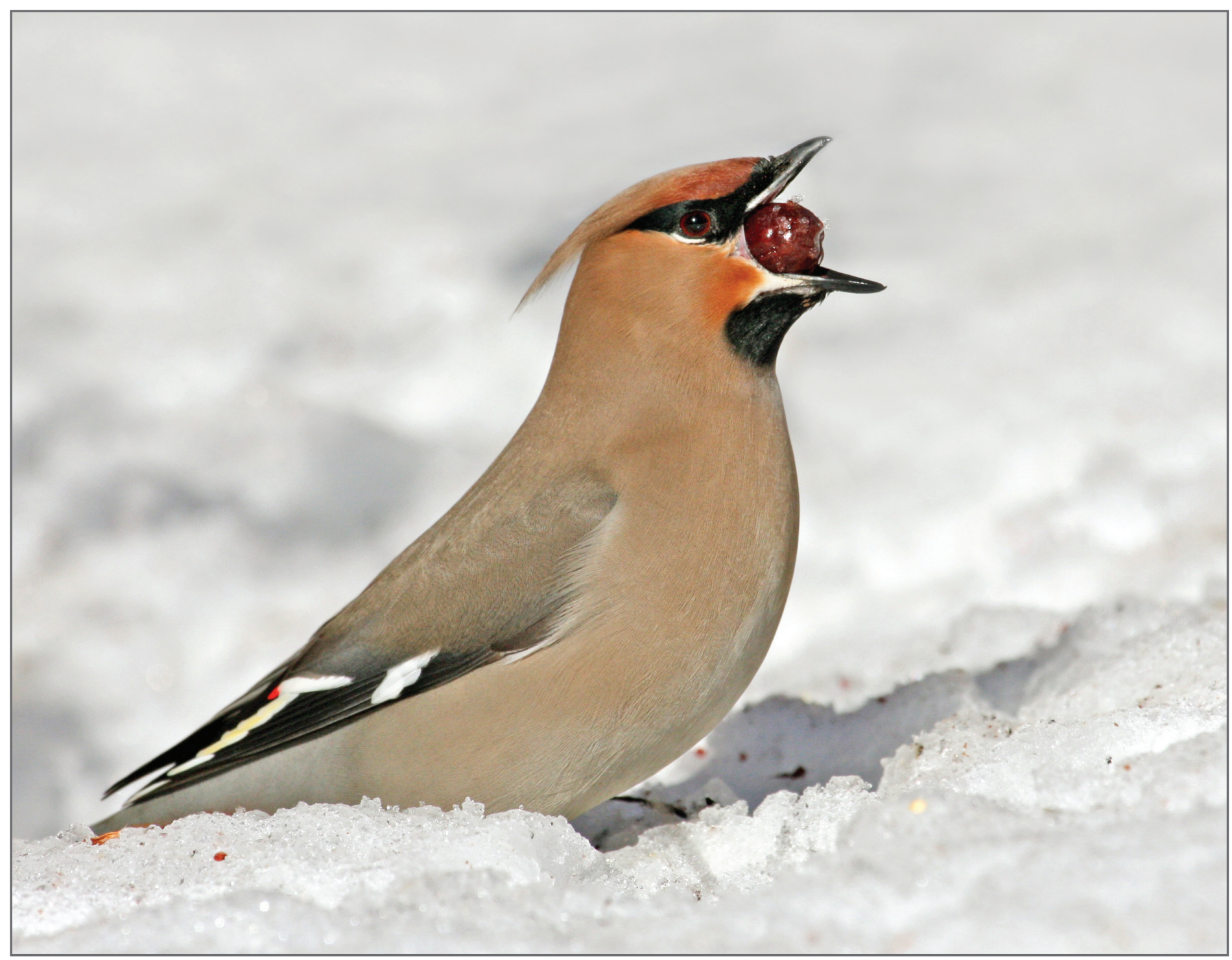

Bohemian Waxwing. Photo credit: Nick Saunders 
65. QU'APPELLE VALLEY DAM.

Andrew Elgin, Joshua Erickson, Nick Saunders, Jan Shadick, Stan Shadick, Carl Siemens, Hollice Siemens, Marten Stoffel, Guy Wapple, Michael Williams.

66. REGINA. Ingrid Alesich, Brian Armstrong, Robert Berthiaume, Lionel Bonneville, Stephane Bonneville, Stephane Canevet, Jared Clarke, Roberta Cox, Lolamae Crawley, Ron Crawley, Kathleen Donauer, Jim Elliot, Ruth Englund, Ian Fallas, Jackie Fauth, Phyl Fauth, Terry Ford, Shirley Friel, Brendan Graham, Chris Harris, JoAnne Harrison, Mark Harrison, Dale Hjertaas, Trevor Herriot, Marie James, Fran Kerbs, Laurie Koepke, Luciel Lipka, Sarah Ludlow, Bob Luterbach, Lauren Mang, Kim Mann, Val Mann, Jim Nordquist, Jens Pedersen, Joseph Poissant, Brett Quiring, Chris Ratch, Serena Rieck, Ed Rodger, Nick Selinger, Brian Sterenberg, Frank Switzer, Mary Switzer, Hanna Walczykowski, Lorraine Weidner, Bradley Yee, Ken Yee.

67. ROKEBY. Marlene Bartlett, Lloyd Liebrecht, Edna Lockhart, Morley Maier, Stephanie Newsham, Lorne Niebergal, Fred Phillips, Martin Phillips, Kirk Screpnick, Ray Thies, Bruce Tulloch, Allan Wheeler, Mitch Wilock.

68. ROSCOMMON S.D. Bernice Althouse, Kate Althouse, Ruby Finnie, Brian Irving, Sophie Jankowski, Joan Lillibo, Dianne Sloan, Graham Sloan, Marguerite Sloan.

69. ROULEAU. Stuart Anderson, Allen McGratten, Noreen McGratten, Patricia Sterzuk.

70. ROUND LAKE (Prince Albert Area) Crystal Frenette.

71. ROUND LAKE (Qu'Appelle Valley). Jaxon Finkas, Boyd Metzler, Mary Ward.

72. SALTCOATS. Arden Bradford, Olga Brygider, Len Cameron, Michelle Easton, Walter Farquharson, Dave Herron, Gloria Herron, Gerri Knudsen, Ron Knudsen, Mardell Mack, Fern McKay, Randy Torrie, Val Trowell, Rob Wilson.
73. SASKATCHEWAN LANDING PROVINCIAL PARK. Glen Pederson, Marten Stoffel, Dan Zazelenchuk.

\section{SASKATCHEWAN RIVER FORKS.} Carman Dodge, Don Weidl.

75. SASKATOON. Joanne Adams, Omar Aschim, Eveline Boudreau, Anne Brander, Delores Burkhart, Jacquie Christenson, Louise Cook, Lorne Duczek, Joshua Erickson, Joan Feather, Lesley Fell, Greg Fenty, Jennifer Fenty, David Forbes, Kyron Giroux, Bob Godwin, Mike Gollop, Donna Goodridge, Jim Goodridge, Allison Hadland, Donna Haughen, Janet Hill, Terri Jackson, Jeff Jensen, Julie Jensen, Ron Jensen, Richard Kerbes, Troy Lange, Don Loran, Janet Loran, Audrey MacKenzie, Bill MacKenzie, Priscilla Mah, Val Martz, Kari McCrea, Sabryna McCrae, Bob McNaughton, Kathy Meeres, Coleen Meldrum, Scott Mitchell, Carol Monahan, Joe Monahan, Jonathan Moore-Wright, Verity Moore-Wright, Hilda Noton, John Patterson, Bill Robertson, Lisa Rohachuk, Marc Sabourin, Craig Salisbury, Lorraine Salisbury, Trish Santo, Scott Saretsky, Nick Saunders, Margie Scharf, Murray Scharf, Jan Shadick, Stan Shadick, Barb Sprigings, Marten Stoffel, Margo Taylor, Phil Taylor, Don Torrie, Guy Wapple, Sandra Wapple, Olive Watts, Trent Watts, George West, Michael Williams, Norman Zlotkin.

76. SAWYER LAKE. Maureen Blight, Kathleen Pitt, Michael Pitt, David Weiman

77. SHAMROCK. Hugh Henry, Sylvia Knudsen, Iris McNeill, Darwin Menke, Joel Priebe, Myrna Priebe, Lori Wilson.

78. SHELL LAKE. Ryan Dudragne, LeeAnn Latremouille, Carole Martin, Kay Willson, Phil Willson.

79. SNOWDEN. Esther Chamberlin, Sonya Fidyk, Gus Fomrades, Diane Friesen, Don Friesen, Ed Hagel, Irene Hagel, Doreen Long, Tom McLean, Linda Patton, Jack Pickett, Valerie Rein Bill Thompson, Muriel Thompson, Tim Thompson, Irene White.
: 80. SPINNEY HILL. Ed Driver.

81. SQUAW RAPIDS. Bert Dalziel, Joan Dalziel, Ryan Dudragne, Clara Weighill.

82. SWIFT CURRENT. Anita Anderson, Jacquie Bolton, Stacy Bolton, Norris Currie, Ryan Dudragne, Laurent Dudragne, Mary Ann Dudragne, Ryan Dudragne, Arnie Ens, Norma Hain, Hugh Henry, Verna Lynn Knipfel, Dot Letkeman, Maxine Lundman, Rita McLaughlin, Kevin Moore, Janet Payne, Harold Steppuhn, Irene Stinson, Sharlane Toole, Kae Waters.

83. THICKWOOD HILLS-SPIRITWOOD. Margie Fast, Giles Lalonde, Susan Lalonde, Carole Martin, Kay Willson, Philip Willson.

84. TURTLE LAKE. David Forbes, Fred Hegelton, Jackie Hegelton, Brent Keen, Cheryl Robbins.

85. TURTLEFORD. Angela Csiki, Hank DeGraaf, Miles Johnson, Brent Keen, Louise Lundberg, Ron Perkins, Richard Roney, Colin Rudolf, Marg Uhlig.

86. WEYBURN. Glen Fleming, Millie Fleming, Dale Huff, Sandy Huff, Charlotte Payak, Cole Payak, Don Payak, Joe Stefaniuk, Doyle Thomas, Tanis Thomas, Val Thomas, Kim Thorson, Myrt Thorson, John Whitell.

87. WHITE BEAR. Greg Nelson, Yvonne Nelson, Glen Pederson, Dan Zazelenchuk.

88. WHITEWOOD. Ken Aldous, Carole Armstrong, Cindy Ashfield, Joe Ashfield, Paul Ashfield, Kerri Bachtold, Joyce Kydd, Sarah Mambourg, Boyd Metzler, Harry Mitchell, Marilyn Mitchell, Donna Mohr, Margaret Niemenen, Paul Niemenen, Tony Saltasuk, Doug Shepherd.

89. WINGARD. Rebecca Beam. 
TABLE 1. Weather and Snow Cover.

\begin{tabular}{|c|c|c|c|c|c|c|c|c|c|}
\hline LOCALITY & DATE & $\begin{array}{l}\text { MIN } \\
\text { TEMP } \\
\text { (C) }\end{array}$ & $\begin{array}{l}\text { MAX } \\
\text { TEMP } \\
\text { (OC) }\end{array}$ & $\begin{array}{c}\text { MIN } \\
\text { WIND } \\
\text { (KM/HR) }\end{array}$ & $\begin{array}{c}\text { MAX } \\
\text { WIND } \\
(\mathrm{KM} / \mathrm{HR})\end{array}$ & $\begin{array}{l}\text { MIN } \\
\text { SMOW } \\
\text { (CM) }\end{array}$ & $\begin{array}{l}\text { MAX } \\
\text { SNOW } \\
\text { (CM) }\end{array}$ & SKY A.M. & SKY P.M. \\
\hline Archerwill & 18 Dec 2017 & -4 & -2 & 16 & 20 & 10 & 10 & clear & clear \\
\hline Avonlea & 3 Jan 2018 & -7 & 0 & 0 & 0 & 0 & 10 & overcast & partly cloudy \\
\hline Balgonie & 30 Dec 2017 & -36 & -28 & 3 & 10 & 3 & 10 & clear & mostly clear \\
\hline Biggar & 14 Dec 2017 & -2 & -1 & 18 & 30 & 0 & 10 & mostly clear & partly cloudy \\
\hline Birch Hills & 4 Jan 2018 & -27 & -18 & 6 & 11 & 14 & 14 & light snow & overcast \\
\hline Borden-Radisson & $2 \operatorname{Jan} 2018$ & -19 & -6 & 4 & 16 & 0 & 3 & partly cloudy & mostly clear \\
\hline Broadview & 21 Dec 2017 & -23 & -9 & 5 & 20 & 0 & 5 & clear & partly cloudy \\
\hline Cater & 4 Jan 2018 & -12 & -9 & 0 & 0 & 6 & 20 & overcast & overcast \\
\hline Chatsworth S.D. & 5 Jan 2018 & -20 & -16 & 10 & 25 & 3 & 20 & cloudy & cloudy \\
\hline Christopher Lake & 18 Dec 2017 & -3 & -1 & 6 & 20 & 10 & 15 & partly cloudy & partly cloudy \\
\hline Churchbridge B & 31 Dec 2017 & & & & & & & & \\
\hline Clark's Crossing & 16 Dec 2017 & -5 & 0 & 15 & 28 & 0 & 20 & partly cloudy & cloudy \\
\hline Coronach & 17 Dec 2017 & -6 & 1 & 16 & 35 & 0 & 2 & mostly clear & cloudy \\
\hline Craven & 16 Dec 2017 & -33 & -22 & 9 & 17 & 2 & 15 & mostly clear & mostly clear \\
\hline Creighton & 16 Dec 2017 & -5 & 1 & 19 & 31 & 0 & 2 & clear & clear \\
\hline Crooked Lake & 18 Dec 2017 & -2 & 0 & 6 & 11 & 0 & 10 & cloudy & partly cloudy \\
\hline Crooked River & 30 Dec 2017 & -35 & -30 & 6 & 19 & 25 & 30 & clear & clear \\
\hline Cypress Hills P.P. & 29 Dec 2017 & -28 & -28 & 20 & 25 & 5 & 20 & mod. Fog & partly cloudy \\
\hline Denholm & 25 Dec 2017 & -28 & -22 & 0 & 12 & 1 & 20 & clear & clear \\
\hline Dorintosh & 26 Dec 2017 & & -23 & & 15 & & 25 & overcast & overcast \\
\hline Dundurn & 4 Jan 2018 & -11 & -7 & 6 & 11 & 4 & 15 & cloudy & cloudy \\
\hline Duval & 1 Jan 2018 & -30 & -21 & 5 & 10 & 3 & 40 & clear & mostly clear \\
\hline Eastend & 4 Jan 2018 & -9 & -2 & 10 & 15 & 5 & 10 & partly cloudy & mostly clear \\
\hline Ebenezer B & 5 Jan 2018 & -9 & -6 & 15 & 15 & 30 & 30 & mostly clear & mostly clear \\
\hline Endeavour & 25 Dec 2017 & -36 & -22 & 0 & 0 & 20 & 40 & mostly clear & overcast \\
\hline Estevan & 4 Jan 2018 & -18 & -10 & 7 & 20 & 0 & 10 & partly cloudy & partly cloudy \\
\hline Estuary North & $2 \operatorname{Jan} 2018$ & -7 & -7 & 5 & 5 & & & clear & clear \\
\hline Fenton & 3 Jan 2018 & -13 & -10 & 20 & 20 & 10 & & overcast & overcast \\
\hline Floral & 28 Dec 2017 & -31 & -22 & 11 & 15 & 0 & 10 & partly cloudy & partly cloudy \\
\hline Fort Qu'Appelle & 16 Dec 2017 & 0 & 2 & 2 & 2 & 0 & 2 & clear & clear \\
\hline Gardiner Dam & 18 Dec 2017 & -7 & -1 & 15 & 41 & 0 & 10 & partly cloudy & mostly clear \\
\hline Good Spirit Lake & 21 Dec 2017 & -22 & -12 & 15 & 30 & 12 & 25 & clear & overcast \\
\hline Grasslands N.P. & 17 Dec 2017 & -7 & -1 & 10 & 20 & 0 & 1 & partly cloudy & light snow \\
\hline Grayson & 29 Dec 2017 & -32 & -28 & 5 & 20 & 0 & 10 & mostly clear & mostly clear \\
\hline Greenwater & 3 Jan 2018 & -17 & -12 & 11 & 11 & 30 & 40 & mod. snow & overcast \\
\hline Grenfell & 3 Jan 2018 & -22 & -14 & 2 & 5 & 2 & 4 & clear & partly cloudy \\
\hline Harris & 21 Dec 2017 & -13 & -10 & 18 & 35 & 0 & 5 & overcast & light snow \\
\hline Hazlet & 27 Dec 2017 & -23 & -20 & 2 & 20 & 0 & 5 & cloudy & partly cloudy \\
\hline Hudson Bay & 5 Jan 2018 & -32 & -25 & 2 & 5 & 20 & 25 & mostly clear & \\
\hline Indian Head & 29 Dec 2017 & -28 & -28 & 0 & 0 & 10 & 10 & light snow & light snow \\
\hline Kenaston & 15 Dec 2017 & 0 & 3 & 0 & 10 & 0 & 1 & mostly clear & overcast \\
\hline Kenosee Lake & 24 Dec 2017 & -23 & -19 & 20 & 30 & 4 & 20 & clear & partly cloudy \\
\hline Ketchen North & 26 Dec 2017 & -33 & -26 & 0 & 10 & 25 & 30 & clear & clear \\
\hline Kindersley North & 26 Dec 2017 & -30 & -29 & 2 & 5 & 0 & 2 & clear & clear \\
\hline Kinloch & $2 \operatorname{Jan} 2018$ & -14 & -11 & 30 & 39 & 22 & 25 & cloudy & partly cloudy \\
\hline
\end{tabular}




\begin{tabular}{|c|c|c|c|c|c|c|c|c|c|}
\hline LOCALITY & DATE & $\begin{array}{l}\text { MIN } \\
\text { TEMP } \\
\text { (C) }\end{array}$ & $\begin{array}{l}\text { MAX } \\
\text { TEMP } \\
\text { (OC) }\end{array}$ & $\begin{array}{c}\text { MIN } \\
\text { WIND } \\
\text { (KM/HR) }\end{array}$ & $\begin{array}{c}\text { MAX } \\
\text { WIND } \\
\text { (KM/HR) }\end{array}$ & $\begin{array}{c}\text { MIN } \\
\text { SMOW } \\
\text { (CM) }\end{array}$ & $\begin{array}{l}\text { MAX } \\
\text { SNOW } \\
\text { (CM) }\end{array}$ & SKY A.M. & SKY P.M. \\
\hline Kyle & $29 \operatorname{Dec} 2017$ & -31 & -28 & 12 & 29 & 0 & 4 & partly cloudy & clear \\
\hline La Ronge & 26 Dec 2017 & -34 & -30 & 4 & 6 & 30 & 30 & clear & clear \\
\hline Leader North & 3 Jan 2018 & -13 & 0 & 0 & 5 & 2 & 5 & mostly clear & overcast \\
\hline Leroy & 2 Jan 2018 & -11 & -9 & 10 & 25 & 0 & 30 & overcast & mostly clear \\
\hline Love-Torch River & 26 Dec 2017 & -31 & -5 & 10 & 15 & 5 & 17 & clear & clear \\
\hline Luseland & 4 Jan 2018 & -9 & -3 & 0 & 10 & 2 & 13 & mostly clear & mostly clear \\
\hline Meadow Lake & 26 Dec 2017 & -30 & -26 & 13 & 26 & & & clear & clear \\
\hline Melfort & $30 \operatorname{Dec} 2017$ & -33 & -28 & 5 & 15 & 5 & 15 & clear & clear \\
\hline Moose Jaw & 4 Jan 2018 & -12 & -8 & 8 & 14 & 0 & 4 & cloudy & partly cloudy \\
\hline Moose Mountain & 4 Jan 2018 & -17 & -15 & 23 & 25 & 2 & 2 & cloudy & light snow \\
\hline Morse & 27 Dec 2017 & -20 & -2 & 11 & 16 & 6 & 18 & overcast & mostly clear \\
\hline Nipawin & 23 Dec 2017 & -25 & -23 & 0 & 9 & 11 & 11 & clear & clear \\
\hline Nisbet Forest NW & $14 \operatorname{Dec} 2017$ & -4 & 0 & 3 & 13 & 14 & 14 & overcast & overcast \\
\hline Nisbet Forest West & 27 Dec 2017 & -25 & -20 & 5 & 10 & 5 & 15 & clear & clear \\
\hline Odessa & 3 Jan 2018 & -30 & -24 & 6 & 29 & & & light snow & light snow \\
\hline Pike Lake & 5 Jan 2018 & -14 & -9 & 0 & 32 & 0 & 20 & clear & clear \\
\hline Ponteix & 26 Dec 2017 & -32 & -24 & 10 & 15 & 2 & 15 & clear & clear \\
\hline Prince Albert & 17 Dec 2017 & -6 & -3 & 5 & 10 & 10 & 10 & overcast & overcast \\
\hline Qu'Appelle & 28 Dec 2017 & -32 & -26 & 6 & 11 & 0 & 10 & clear & clear \\
\hline Qu'Appelle Dam & 17 Dec 2017 & -6 & 1 & 10 & 21 & 0 & 10 & cloudy & partly cloudy \\
\hline Regina & 26 Dec 2017 & -32 & -25 & 9 & 18 & 3 & 10 & clear & clear \\
\hline Rokeby & 19 Dec 2017 & -10 & & 2 & & 5 & 10 & mostly clear & mostly clear \\
\hline Roscommon S.D. & 3 Jan 2018 & -24 & -13 & 20 & 25 & 10 & 15 & cloudy & overcast \\
\hline Rouleau & 18 Dec 2017 & 0 & 3 & 1 & 6 & 0 & 1 & cloudy & mostly clear \\
\hline Round Lake (P.A.) & & -14 & -12 & 0 & 13 & 6 & 8 & overcast & \\
\hline Round Lake (Q.V.) & 16 Dec 2017 & 0 & 1 & 6 & 11 & 0 & 15 & clear & mostly clear \\
\hline Saltcoats & 15 Dec 2017 & -5 & -5 & 12 & 20 & 14 & 14 & & mod. Snow \\
\hline Sask. Landing P.P. & 28 Dec 2017 & -28 & -18 & 6 & 19 & 2 & 5 & clear & cloudy \\
\hline Sask. River Forks & 16 Dec 2017 & -9 & -2 & 5 & 10 & 5 & 10 & partly cloudy & cloudy \\
\hline Saskatoon & 26 Dec 2017 & -37 & -25 & 9 & 15 & 0 & 20 & clear & clear \\
\hline Sawyer Lake & 28 Dec 2017 & -27 & -22 & 4 & 7 & 30 & 40 & clear & clear \\
\hline Shamrock & 14 Dec 2017 & -4 & -1 & 5 & 30 & 0 & 0 & clear & clear \\
\hline Shell Lake & 14 Dec 2017 & -2 & -1 & 0 & 20 & 0 & 15 & overcast & partly cloudy \\
\hline Snowden & $3 \operatorname{Jan} 2018$ & -20 & -19 & 30 & 39 & 5 & 23 & cloudy & cloudy \\
\hline Spinney Hill & 16 Dec 2017 & -7 & -3 & 20 & 40 & 0 & 10 & clear & partly cloudy \\
\hline Squaw Rapids & 5 Jan 2018 & -18 & -15 & 10 & 18 & 10 & 30 & light snow & light snow \\
\hline Swift Current & 16 Dec 2017 & -7 & -1 & 20 & 30 & 0 & 1 & mostly clear & mostly clear \\
\hline $\begin{array}{l}\text { Thickwood- } \\
\text { Spiritwood }\end{array}$ & 15 Dec 2017 & 0 & 3 & 10 & 25 & 0 & 10 & overcast & light rain \\
\hline Turtle Lake & 17 Dec 2017 & -7 & -4 & 2 & 5 & 8 & 20 & cloudy & clear \\
\hline Turtleford & 30 Dec 2017 & -36 & -29 & 0 & 7 & 1 & 30 & mostly clear & mostly clear \\
\hline Weyburn & 4 Jan 2018 & -19 & -15 & 16 & 20 & 1 & 2 & cloudy & cloudy \\
\hline White Bear & 21 Dec 2017 & -12 & -7 & 12 & 19 & 0 & 1 & cloudy & overcast \\
\hline Whitewood & 27 Dec 2017 & -31 & -20 & 20 & 39 & 4 & 10 & overcast & partly cloudy \\
\hline Wingard & 3 Jan 2018 & & & & & & & & \\
\hline AVERAGES & & -18 & -12 & 9 & 18 & 6 & 14 & & \\
\hline
\end{tabular}


TABLE 2. Effort and Habitat Coverage. Wild Fruit: $p=p o o r, f=f a i r, g=g o o d, e=e x c e l l e n t$.

\begin{tabular}{|c|c|c|c|c|c|c|c|c|c|c|c|c|c|c|c|c|c|c|c|c|}
\hline LOCALITY & 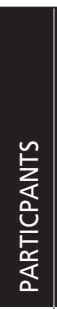 & $\begin{array}{l}\text { - } \\
\text { 인 } \\
z \\
0 \\
\sum \\
\underline{z}\end{array}$ & 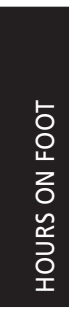 & 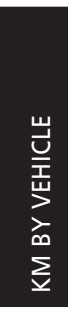 & 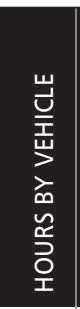 & 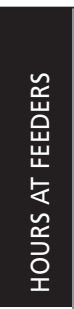 & 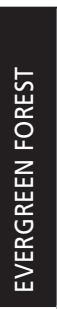 & 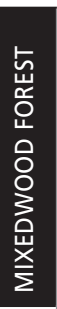 & 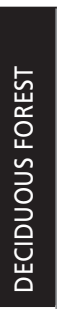 & 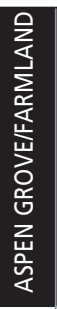 & 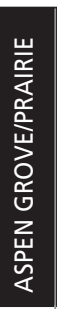 & 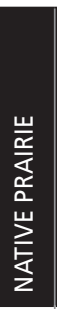 & 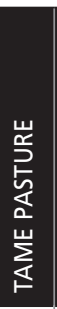 & 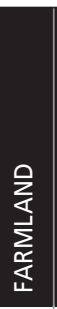 & 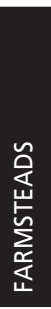 & 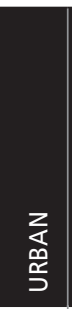 & $\begin{array}{l}\text { 営 } \\
\vdots \\
\vdots \\
\text { ż } \\
\text { o }\end{array}$ & 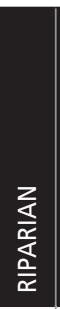 & 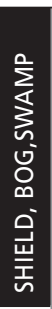 & 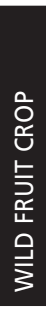 \\
\hline Archerwill & 14 & 3.2 & 1.5 & 0 & 0.0 & 8.0 & & 25 & & & & & & 50 & 25 & & & & & $p$ \\
\hline Avonlea & 4 & 0.2 & 0.3 & 74 & 3.5 & 4.0 & & & 5 & & & 5 & 5 & 40 & 5 & 40 & & & & $\mathrm{p}$ \\
\hline Balgonie & 10 & 8.0 & 0.5 & 206 & 12.0 & 4.0 & & & & 30 & & & & 40 & & 30 & & & & $f$ \\
\hline Biggar & 11 & 13.0 & 11.0 & 390 & 11.5 & 5.0 & & & & 20 & & & & 40 & 5 & 35 & & & & $p$ \\
\hline Birch Hills & 2 & 1.0 & 1.3 & 114 & 2.5 & 4.0 & & & 5 & 15 & 5 & & 5 & 55 & 5 & 5 & & 5 & & g \\
\hline Borden-Radisson & 5 & 5.0 & 2.5 & 145 & 5.5 & 2.0 & & & & & & 10 & & 65 & 5 & 15 & & 5 & & $f$ \\
\hline Broadview & 4 & 1.0 & 1.0 & 135 & 5.5 & 2.0 & & & 20 & 25 & & 5 & 10 & 25 & 5 & 10 & & & & $\mathrm{p}$ \\
\hline Cater & 2 & 0.0 & 0.0 & 243 & 7.0 & 1.6 & 5 & 10 & 5 & 15 & & & 5 & 50 & 5 & 5 & & & & $f$ \\
\hline Chatsworth S.D. & 11 & 1.0 & 10.0 & 75 & 4.0 & 5.0 & & & & 80 & & 10 & 5 & & 5 & & & & & $p$ \\
\hline Christopher Lake & 1 & 12.0 & 2.5 & 0 & 0.0 & 0.0 & & 60 & & 30 & & & & & & 10 & & & & $p$ \\
\hline Churchbridge B & 1 & & & & & 1.0 & & & & & & & & & & 100 & & & & $\mathrm{p}$ \\
\hline Clark's Crossing & 24 & 13.5 & 14.3 & 507 & 26.0 & 3.5 & & & & 28 & 4 & 4 & 4 & 22 & 10 & 22 & 1 & 5 & & $\mathrm{p}$ \\
\hline Coronach & 1 & 0.8 & 0.4 & 221 & 7.3 & 0.0 & & & & & & 15 & 15 & 55 & $<1$ & 10 & 5 & & & e \\
\hline Craven & 40 & 19.3 & 21.9 & 318 & 21.9 & 8.0 & & & & 30 & 15 & 5 & 5 & 20 & 10 & 15 & & & & $f$ \\
\hline Creighton & 5 & 9.0 & 5.0 & 203 & 9.0 & 8.0 & 20 & 10 & & & & & & & & 40 & & & 30 & g \\
\hline Crooked Lake & 1 & 1.0 & 0.3 & 139 & 4.5 & 0.0 & & & 25 & 20 & & & & 20 & & 20 & 15 & & & $\mathrm{p}$ \\
\hline Crooked River & 2 & 0.0 & 0.0 & 0.0 & 0.0 & 8.0 & & 40 & & & & & & 40 & 20 & & & & & g \\
\hline Cypress Hills P.P. & 3 & 4.0 & 5.0 & 18 & 4.0 & 0.0 & 40 & 40 & 8 & & & 10 & & & 2 & & & & & g \\
\hline Denholm & 5 & 0.0 & 0.0 & 240 & 7.5 & 5.0 & & & & 10 & 5 & & 5 & 70 & 5 & 5 & & & & g \\
\hline Dorintosh & 4 & 20.0 & 8.0 & 20 & 0.5 & 0.0 & & 60 & & & & & & & 40 & & & & & $p$ \\
\hline Dundurn & 4 & 8.0 & 2.0 & 160 & 5.8 & 0.0 & & & & & & 25 & & 55 & 10 & 10 & & & & $p$ \\
\hline Duval & 2 & 2.0 & 1.5 & 117 & 4.5 & 0.5 & & & & 15 & 15 & & & 40 & 10 & 20 & & & & $f$ \\
\hline Eastend & 15 & 7.0 & 9.0 & 180 & 33.0 & 2.5 & & 5 & & 20 & 50 & & 5 & 5 & & 15 & $<1$ & & & g \\
\hline Ebenezer B & 1 & 0.0 & 0.0 & 48 & 5,5 & 2.0 & & 10 & & & & 30 & 35 & 15 & & 5 & & 5 & & $p$ \\
\hline Endeavour & 1 & 0.0 & 0.0 & 35 & 2.0 & 4.0 & & & & 60 & & & & & 20 & 20 & & & & $\mathrm{p}$ \\
\hline Estevan & 8 & 5.0 & 4.3 & 197 & 4.8 & 5.0 & & & & & & & & 20 & 4 & 16 & 10 & 50 & & $\mathrm{p}$ \\
\hline Estuary North & 3 & 3.0 & 3.0 & 20 & 4.0 & 4.0 & & & 10 & & & 40 & & 40 & 10 & & & & & $f$ \\
\hline Fenton & 1 & 0.0 & 0.0 & 80 & 3.2 & 0.0 & & & & & & & & & & & & & & $p$ \\
\hline Floral & 2 & 4.0 & 2.5 & 170 & 6.0 & 1.0 & & & & 20 & & & & 60 & 5 & 15 & & & & $p$ \\
\hline Fort Qu'Appelle & 20 & 0.0 & 0.0 & 200 & 20.0 & 0.0 & & & & 25 & 20 & & 10 & 20 & 5 & 20 & & & & $p$ \\
\hline Gardiner Dam & 12 & 19.0 & 18.3 & 419 & 15.8 & 1.0 & & & & 5 & & & & 50 & 6 & 10 & 29 & & & $\mathrm{p}$ \\
\hline Good Spirit Lake & 3 & 0.0 & 0.0 & 120 & 6.0 & 0.0 & & & & 30 & & & 40 & & 5 & 25 & & & & $f$ \\
\hline Grasslands N.P. & 1 & 2.0 & 0.5 & 290 & 8.5 & 0.0 & & & & & & 90 & & & 5 & & 5 & & & $f$ \\
\hline Grayson & 4 & 1.0 & 0.5 & 110 & 5.5 & 6.0 & & & & 35 & 20 & & & 20 & 15 & 5 & & 5 & & \\
\hline Greenwater & 3 & 4.0 & 2.0 & 93 & 5.0 & 8.0 & & 54 & & & & & & 40 & 2 & 4 & & & & $p$ \\
\hline Grenfell & 3 & 0.0 & 0.0 & 130 & 6.0 & 2.5 & & & 15 & 15 & & 5 & 15 & 25 & 10 & 15 & & & & $\mathrm{p}$ \\
\hline Harris & 4 & 8.0 & 6.8 & 172 & 6.3 & 0.0 & & & & 2 & & & & 50 & 6 & 42 & & & & $\mathrm{p}$ \\
\hline Hazlet & 1 & 1.1 & 0.6 & 276 & 7.2 & 0.0 & & & & & $<1$ & 10 & 15 & 65 & 5 & 5 & & & & $\mathrm{p}$ \\
\hline Hudson Bay & 5 & 0.0 & 0.0 & 0 & 0.0 & 7.0 & & 40 & 40 & & & & & & 20 & & & & & $f$ \\
\hline Indian Head & 21 & 1.5 & 1.0 & 262 & 5.0 & 25.0 & 5 & & & 10 & & & & 75 & 5 & 5 & & & & $p$ \\
\hline Kenaston & 3 & 0.0 & 0.0 & 84 & 4.0 & 1.0 & & & & & & & & 90 & 5 & 5 & & & & \\
\hline Kenosee Lake & 2 & 2.0 & 1.0 & 154 & 3.0 & 1.0 & & & 20 & 25 & 10 & & 5 & & & 40 & & & & $\mathrm{p}$ \\
\hline Ketchen North & 1 & 1.0 & 1.0 & 50 & 2.5 & 6.0 & & & & 30 & & & 20 & 20 & 20 & 10 & & & & \\
\hline Kindersley North & 2 & 0.0 & 0.0 & 87 & 4.5 & 0.0 & & & & & & & & & & & & & & \\
\hline Kinloch & 7 & 3.0 & 1.0 & 117 & 13.0 & 4.0 & & 10 & 20 & 30 & & & & 20 & 20 & & & & & $p$ \\
\hline
\end{tabular}




\begin{tabular}{|c|c|c|c|c|c|c|c|c|c|c|c|c|c|c|c|c|c|c|c|c|}
\hline LOCALITY & 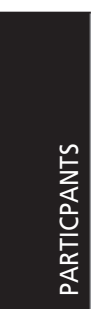 & 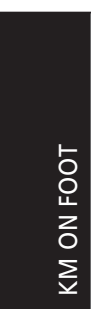 & $\begin{array}{l}\text { ㅇ } \\
\text { 운 } \\
\text { zo } \\
\text { w } \\
\text { 옥 } \\
\text { 오 }\end{array}$ & 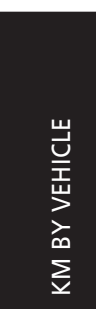 & 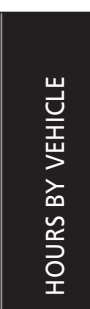 & 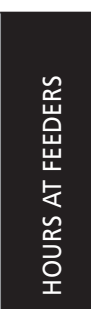 & 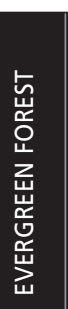 & 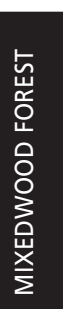 & 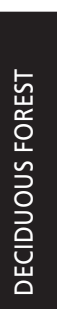 & 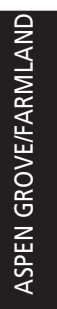 & 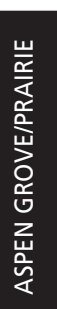 & 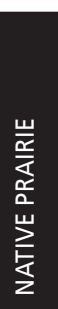 & 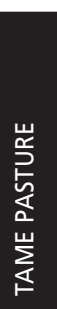 & 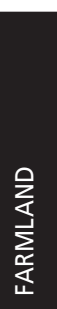 & 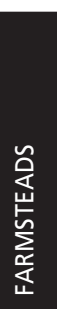 & $\begin{array}{l}z \\
\frac{1}{\alpha} \\
\stackrel{y}{s}\end{array}$ & $\begin{array}{l}\text { 岕 } \\
\frac{\mathrm{t}}{3} \\
z \\
\text { z } \\
0\end{array}$ & 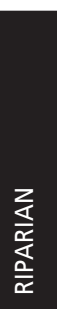 & 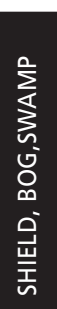 & 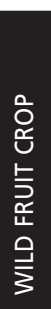 \\
\hline Kyle & 4 & 5.0 & 2.0 & 325 & 12.0 & 0.0 & & & & & & 15 & 5 & 30 & 20 & 15 & & 15 & & $p$ \\
\hline La Ronge & 10 & 5.0 & 8.0 & 75 & 5.5 & 16.5 & 20 & & 10 & & & & & 10 & & 50 & & 10 & & $p$ \\
\hline Leader North & 1 & 4.0 & 1.0 & 50 & 3.0 & 3.5 & & & & & & 40 & 20 & 20 & 20 & & & & & $f$ \\
\hline Leroy & 3 & 3.0 & 1.5 & 148 & 5.0 & 0.5 & & & & 35 & & & & 35 & 10 & 20 & & & & $g$ \\
\hline Love-Torch River & 26 & 1.0 & 0.5 & 348 & 14.5 & 15.0 & 20 & 10 & & 50 & & & & & 10 & 10 & & & & $p$ \\
\hline Luseland & 6 & 9.0 & 4.5 & 372 & 5.5 & 2.0 & & & & 40 & 10 & 5 & 5 & 20 & 10 & 10 & & & & $f$ \\
\hline Meadow Lake & 2 & 0.0 & 0.0 & 133 & 3.0 & 0.0 & & 50 & & & & & & 30 & & 20 & & & & $f$ \\
\hline Melfort & 9 & 0.5 & 0.5 & 210 & 12.0 & 6.0 & & & & 5 & & & & 45 & & 50 & & & & $\mathrm{p}$ \\
\hline Moose Jaw & 4 & 14.1 & 5.7 & 331 & 11.2 & 0.0 & & & 5 & & & 5 & 10 & 35 & & 35 & & 10 & & $f$ \\
\hline Moose Mountain & 7 & 0.0 & 0.0 & & 5.0 & 0.5 & & 60 & & & & 2 & & 29 & 3 & 6 & & & & $f$ \\
\hline Morse & 10 & 4.0 & 1.3 & 496 & 22.5 & 0.0 & & & & & & & 3 & 90 & 7 & & & & & $\mathrm{~g}$ \\
\hline Nipawin & 17 & 2.0 & 1.0 & 163 & 10.0 & 16.0 & 15 & 5 & & 15 & & & & 20 & 10 & 30 & 5 & & & $\mathrm{p}$ \\
\hline Nisbet Forest NW & 1 & 0.0 & 0.0 & 0 & 0.0 & 4.5 & & 30 & & & & & & 10 & 50 & & & 10 & & $\mathrm{p}$ \\
\hline Nisbet Forest West & 3 & 0.5 & 1.0 & 46 & 2,2 & 3.0 & 25 & 15 & 10 & 30 & & & & 10 & 10 & & & & & $f$ \\
\hline Odessa & 3 & & 3.0 & & 12.0 & 3.0 & & & & 20 & 20 & 10 & 20 & 10 & 20 & & & & & $\mathrm{p}$ \\
\hline Pike Lake & 20 & 27.0 & 18.3 & 383 & 21.8 & 10.0 & & & & & & & & & & & & & & $\mathrm{~g}$ \\
\hline Ponteix & 1 & 0.8 & 0.5 & 221 & 6.5 & 1.1 & & & & $<1$ & & 10 & 20 & 60 & 5 & 5 & $<1$ & $<1$ & & $p$ \\
\hline Prince Albert & 23 & 24.2 & 13.8 & 545 & 29.8 & 4.3 & & & & & & & & & & & & & & $p$ \\
\hline Qu'Appelle & 9 & 3.0 & 1.8 & 191 & 9.5 & 22.0 & & & & 60 & 5 & & 5 & 20 & 5 & 5 & & & & $\mathrm{p}$ \\
\hline Qu'Appelle Dam & 10 & 11.0 & 8.5 & 578 & 19.3 & 0.0 & & & & 31 & 20 & 1 & 2 & 5 & 9 & 18 & 9 & 5 & & $\mathrm{p}$ \\
\hline Regina & 49 & 60.1 & 29.3 & 583 & 26.2 & 14.1 & & & & 5 & & & & 25 & 5 & 65 & & & & $f$ \\
\hline Rokeby & 13 & 0.0 & 0.0 & 140 & 7.5 & 15.0 & & 2 & & & & & & & 95 & 3 & & & & \\
\hline Roscommon S.D. & 9 & 0.0 & 0.0 & 96 & 3.2 & 21.0 & & & & 20 & & & & 60 & 20 & & & & & $p$ \\
\hline Rouleau & 4 & 1.0 & 0.5 & 193 & 5.5 & 1.5 & & & & & 5 & & & 80 & 5 & 10 & & & & $\mathrm{p}$ \\
\hline Round Lake (P.A.) & 1 & 0.0 & 0.0 & 0 & 0.0 & 3.0 & & 30 & & 25 & & & 30 & & & 5 & & 10 & & $\mathrm{p}$ \\
\hline Round Lake (Q.V.) & 3 & 0.0 & 0.5 & 167 & 5.0 & 0.0 & & & 20 & 30 & & & & 20 & & 20 & 10 & & & $p$ \\
\hline Saltcoats & 14 & 0.0 & 0.0 & 79 & 4.0 & 0.0 & & & & 65 & & & & & 20 & 15 & & & & $\mathrm{p}$ \\
\hline Sask. Landing P.P. & 2 & 4.0 & 2.0 & 225 & 7.0 & 0.0 & & & & 5 & & 10 & & 45 & 15 & & & 25 & & $p$ \\
\hline Sask. River Forks & 2 & 1.0 & 0.5 & 89 & 5.3 & 0.5 & 15 & 20 & 15 & 15 & & & 5 & 15 & 5 & & 10 & & & $\mathrm{p}$ \\
\hline Saskatoon & 69 & 72.0 & 30.0 & 924 & 66.0 & 54.0 & 1 & 2 & & 12 & 3 & 1 & 1 & 6 & 2 & 67 & 1 & 4 & & $p$ \\
\hline Sawyer Lake & 4 & 16.0 & 6.0 & 132 & 10.0 & 4.0 & & 20 & 20 & 30 & 25 & & & & 5 & & & & & $f$ \\
\hline Shamrock & 6 & 2.5 & 2.5 & 340 & 12.0 & 0.5 & & & & & & 5 & 5 & 70 & 15 & 5 & & & & \\
\hline Shell Lake & 5 & 5.3 & 2.85 & 238 & 7.5 & 0 & 5 & $<1$ & 25 & 5 & 5 & 5 & 30 & 15 & $<1$ & 5 & & 5 & & $f$ \\
\hline Snowden & 16 & 1.0 & 3.0 & 150 & 3.0 & 7.0 & & 10 & & & & & & 35 & 50 & 5 & & & & $f$ \\
\hline Spinney Hill & 1 & 2.0 & 0.4 & 153 & 5.6 & 0.0 & & & & & & & & & & & & & & $p$ \\
\hline Squaw Rapids & 4 & 1.1 & 1.5 & 179 & 8.2 & 1.5 & & 70 & 20 & & & & & 5 & & & 5 & & & $p$ \\
\hline Swift Current & 20 & 37.9 & 20.0 & 337 & 19.0 & 11.5 & & & & & & & 20 & 5 & 70 & & & 5 & & $f$ \\
\hline $\begin{array}{l}\text { Thickwood- } \\
\text { Spiritwood }\end{array}$ & 5 & 1.5 & 1.0 & 152 & 5.5 & 5.0 & 5 & 10 & & 25 & & & 10 & 30 & 5 & 10 & & 5 & & $g$ \\
\hline Turtle Lake & 5 & 4.0 & 4.0 & 32 & 2.0 & 0.3 & 25 & 25 & & 25 & & & & 25 & & & & & & $f$ \\
\hline Turtleford & 9 & 10.0 & 5.0 & 255 & 8.0 & 10.0 & & & & 45 & & & & 50 & & 5 & & & & $\mathrm{~g}$ \\
\hline Weyburn & 14 & 0.0 & 0.0 & 300 & 11.0 & 4.0 & & & 2 & & 2 & 5 & 6 & 60 & 10 & 15 & & & & $f$ \\
\hline White Bear & 4 & 3.0 & 1.5 & 446 & 12.5 & 0.0 & & & & & & 5 & 5 & 70 & 10 & & & 10 & & \\
\hline Whitewood & 16 & & 0.5 & 303 & 13.5 & 29.0 & & & & 10 & & & & 10 & 10 & 70 & & & & $p$ \\
\hline Wingard & 1 & & & & & & & & & & & & & & & & & & & \\
\hline Totals & 724.0 & 520.0 & 324.1 & 16744.2 & 762.9 & 439.3 & & & & & & & & & & & & & & \\
\hline
\end{tabular}


TABLE 3. Species found on 6 or more counts; ()$=$ seen during count period (CP).

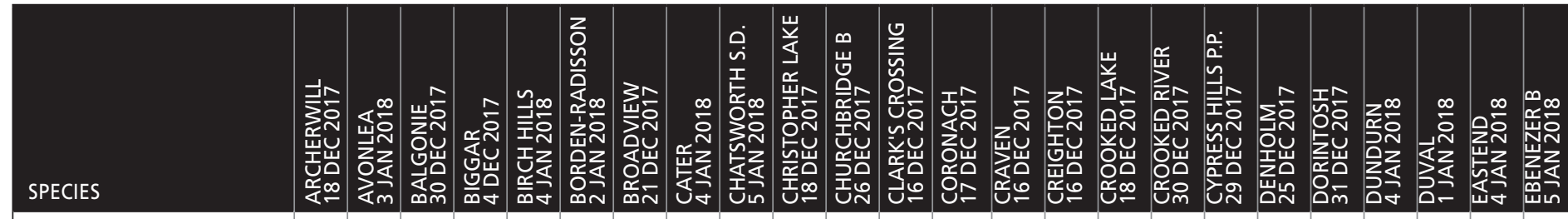

\begin{tabular}{|c|c|c|c|c|c|c|c|c|c|c|c|c|c|c|c|c|c|c|c|c|c|c|c|c|}
\hline Canada Goose & & & & & & & & & & & & 350 & 14855 & & 1 & 602 & & & & & & & & \\
\hline Mallard & & & & & & & & & & & & & 5679 & & & 5 & & & & & & & 3 & \\
\hline Common Goldeneye & & & & & & & & & & & & 26 & 4 & & & & & & & & & & & \\
\hline Gray Partridge & & & 4 & & & 15 & & & & & & 84 & 14 & & & & (5) & & (7) & & 21 & & 6 & \\
\hline Ring-necked Pheasant & & & & & & & & & & & & & 16 & & & & & & & & & & 33 & \\
\hline Ruffed Grouse & 2 & & & & 2 & & 2 & 4 & 5 & & & & & & & & 3 & 1 & (1) & 3 & & & & \\
\hline Sharp-tailed Grouse & (1) & 15 & 24 & 7 & & & 19 & 3 & & & & 40 & 13 & & 3 & & & & (10) & & 17 & 4 & 83 & \\
\hline Rock Pigeon & (10) & 177 & 158 & 68 & 76 & 25 & 5 & 16 & 30 & & & 282 & 78 & 66 & 53 & 66 & & & 89 & 13 & 10 & 73 & 13 & \\
\hline Eurasian Collared-Dove & & 45 & 10 & 13 & & 1 & 2 & & & & & & 25 & 6 & & & & & 4 & & & 1 & 35 & \\
\hline Mourning Dove & & & 1 & 3 & & & & & & & & 1 & & 1 & & & & & & & & & & \\
\hline Bald Eagle & & & 1 & & & & (1) & & & & & 2 & & 2 & & 2 & & & (1) & & & & (1) & 1 \\
\hline Sharp-shinned Hawk & & & & & & & & & & & & 2 & & & & & & & & & & & & \\
\hline Northern Goshawk & & & & & & & & & & & & (1) & & 1 & & & & & (1) & & & & 1 & \\
\hline Rough-legged Hawk & & & & & & & (1) & & & & & & 2 & 1 & & 1 & & & & & & & & \\
\hline Golden Eagle & & & & & & & & & & & & & 2 & & & 1 & & & & & & & 1 & \\
\hline Great Horned Owl & 1 & & 1 & 6 & & & (1) & & (1) & & & 3 & & 1 & & & & & 1 & & 1 & 1 & (1) & \\
\hline Snowy Owl & & 2 & 2 & 1 & & & 1 & & & & & 2 & 3 & 1 & & & (1) & 1 & 4 & & 4 & & & \\
\hline Downy Woodpecker & 4 & & 10 & 4 & 8 & 7 & 3 & 6 & 6 & (2) & 2 & 15 & & 21 & 1 & & 3 & & 6 & 2 & 10 & 5 & 11 & 2 \\
\hline Hairy Woodpecker & 5 & & 5 & 6 & 3 & 4 & 1 & 4 & 7 & 1 & 2 & 10 & & 11 & 3 & & 2 & & 2 & 2 & 3 & & 4 & \\
\hline Black-backed Woodpecker & & & & & & & & (1) & & & & & & & & & & & & & & & & \\
\hline Northern Flicker & & & & & & & & & & & & 1 & 1 & & & & & & & & 3 & & 6 & \\
\hline Pileated Woodpecker & (1) & & & & & 1 & & & & (1) & & & & & 2 & & & & (1) & & & & & \\
\hline Merlin & 1 & & & 1 & & & 1 & & & & & 3 & 1 & & & & & & & & & & & \\
\hline Prairie Falcon & & & & & & & 1 & & & & & & & & & & & & & & 1 & & & \\
\hline Northern Shrike & & & & & & & 2 & & & & & 2 & & 1 & & 2 & & & & & 1 & & & \\
\hline Gray Jay & 1 & & & & & & & & & & & & & & 8 & & & & & 2 & & & & \\
\hline Blue Jay & 26 & 1 & 2 & 3 & 1 & 3 & & 10 & 5 & 7 & & 17 & & 40 & & 2 & 4 & & (2) & 8 & 1 & 1 & 3 & 2 \\
\hline Black-billed Magpie & 7 & 5 & 72 & 129 & 15 & 45 & 26 & 40 & 21 & & 5 & 272 & 4 & 113 & 12 & 21 & 4 & 10 & 83 & 2 & 69 & 25 & 78 & 28 \\
\hline Common Raven & 13 & 2 & 26 & 62 & 6 & 41 & 19 & 83 & 25 & 3 & & 171 & & 61 & 160 & 25 & 1 & 1 & 21 & 4 & 35 & 13 & 22 & 19 \\
\hline Horned Lark & (350) & 20 & & 1 & & & (12) & & & & & & 2 & 2 & & & & & & & & & & \\
\hline Black-capped Chickadee & 41 & & 96 & 84 & 31 & 64 & 23 & 40 & 59 & 44 & 25 & 166 & & 175 & 23 & 48 & 11 & 34 & 10 & 20 & 43 & 24 & 56 & 11 \\
\hline Boreal Chickadee & & & & & & & & 1 & & 3 & & & & & 8 & & & & & & & & & \\
\hline Red-breasted Nuthatch & 1 & (1) & & 6 & & 1 & 1 & & & (2) & & 11 & & 5 & 3 & & & 5 & & 2 & 1 & 1 & 7 & \\
\hline White-breasted Nuthatch & 4 & & 4 & 3 & 5 & 4 & 2 & 6 & 6 & 5 & 1 & 8 & & 26 & & 4 & 2 & & & 2 & 4 & & 1 & \\
\hline Brown Creeper & & & & 1 & & 1 & & & & & & 1 & & & & & & & & & 1 & & & \\
\hline Golden-crowned Kinglet & & & & 6 & & & & & & & & & & & & & & & & & & & & \\
\hline American Robin & & & & 1 & & & & & & & & 1 & & & & & & & & & 2 & & & \\
\hline Varied Thrush & & & & 1 & & & & & & & & 1 & & & & & & & & & & & & \\
\hline European Starling & & 25 & & 36 & & & & & 25 & & & 303 & 16 & 120 & & & & & 7 & & 3 & & & \\
\hline Bohemian Waxwing & (3) & & (1) & 25 & & 24 & 21 & & & & & 34 & & (1) & 76 & & & 15 & 7 & & 432 & 18 & (30) & \\
\hline House Sparrow & & 100 & 240 & 463 & 68 & 240 & 75 & 34 & 104 & & 1 & 1009 & 567 & 451 & 52 & 3 & & 8 & 75 & & 227 & 371 & 157 & 25 \\
\hline Evening Grosbeak & 26 & & & & & & & & & (8) & & & & & 21 & & 2 & & & 5 & & 5 & & \\
\hline Pine Grosbeak & 66 & & & 16 & 13 & 5 & 29 & 95 & 29 & 7 & 6 & 34 & & 5 & 96 & 11 & 6 & (8) & 14 & 10 & 14 & & & \\
\hline House Finch & & 6 & 1 & 41 & & & (1) & & & & & 144 & & 10 & & & & & & & 32 & & 23 & \\
\hline Purple Finch & & & & & & & & & 20 & & & & & & & & & & 1 & & & & & \\
\hline Common Redpoll & 52 & 65 & 291 & 159 & 72 & 62 & 742 & 224 & 176 & 12 & 25 & 121 & 94 & 285 & 71 & 24 & 6 & (2) & 142 & 17 & 320 & 11 & 175 & \\
\hline Hoary Redpoll & 11 & & & 1 & 1 & & (1) & & & & & 2 & & & 6 & & 7 & & 2 & & 1 & & & \\
\hline White-winged Crossbill & & & & & & 9 & & & & & & 5 & & 10 & & & & & & & & & (1) & \\
\hline Pine Siskin & & (1) & & & & & & & & & & 7 & & & & & & & & & & & 6 & \\
\hline American Goldfinch & & & & & & & (1) & & & & & 2 & & (1) & & & & & & & & & & \\
\hline Snow Bunting & (50) & & 9 & 3 & 125 & 550 & & 90 & 32 & & & 58 & & & & & & & 2 & & & 52 & & 100 \\
\hline White-throated Sparrow & & & & & & & & & & & & & & & 1 & & & & & & & & 1 & \\
\hline Dark-eyed Junco & 3 & 2 & 2 & 2 & & & 3 & 2 & 1 & & & 1 & & 2 & 1 & & & & & & 1 & & 3 & \\
\hline Red-winged Blackbird & & & & 1 & & & & & & & & & & & & & & & & & & & & \\
\hline Rusty Blackbird & & 1 & & & & & & & & & & & & & & & & & & & 2 & & & \\
\hline Common Grackle & & 1 & & & & & & & & & & & & & & & & & & & & & & \\
\hline TOTAL BIRDS COUNT DAY & 264 & 468 & 959 & 1242 & 426 & 1102 & 978 & 658 & 551 & 82 & 67 & 3193 & 21441 & 1420 & 619 & 821 & 51 & 75 & 470 & 92 & 1260 & 605 & 747 & 188 \\
\hline TOTAL BIRDS ONLY IN CP & 415 & 2 & 1 & 0 & 0 & 0 & 128 & 1 & 2 & 13 & 0 & 1 & 0 & 2 & 0 & 0 & 6 & 10 & 23 & 0 & 0 & 0 & 33 & 0 \\
\hline TOTAL SPECIES COUNT DAY & 17 & 16 & 20 & 32 & 14 & 19 & 20 & 16 & 16 & 8 & 8 & 38 & 22 & 27 & 23 & 17 & 12 & 8 & 17 & 14 & 28 & 15 & 24 & 8 \\
\hline TOTAL SPECIES ONLY IN CP & 6 & 2 & 1 & 0 & 0 & 0 & 8 & 1 & 2 & 4 & 0 & 0 & 0 & 2 & 0 & 0 & 2 & 2 & 7 & 0 & 0 & 0 & 4 & 0 \\
\hline
\end{tabular}




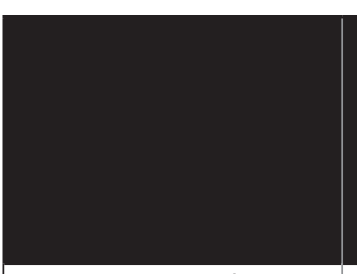

Canada Goose
Mallard

Common Goldeneye Gray Partridge

Ring-necked Pheasant Ruffed Grouse

Sharp-tailed Grouse Rock Pigeon

Eurasian Collared-Dove Mourning Dove Bald Eagle

Sharp-shinned Hawk

Northern Goshawk

Rough-legged Hawk Golden Eagle

Great Horned Owl Snowy Owl

Downy Woodpecker

Hairy Woodpecker Black-backed Woodpecker Northern Flicker

Pileated Woodpecker Merlin Prairie Falcon Northern Shrike Gray Jay Blue Jay Black-billed Magpie Common Raven Horned Lark

Black-capped Chickadee Boreal Chickadee

Red-breasted Nuthatch White-breasted Nuthatch Brown Creeper

Golden-crowned Kinglet American Robin Varied Thrush European Starling

Bohemian Waxwing House Sparrow Evening Grosbeak Pine Grosbeak House Finch Purple Finch

Common Redpoll Hoary Redpoll White-winged Crossbill Pine Siskin

American Goldfinch Snow Bunting White-throated Sparrow
Dark-eyed Junco

Red-winged Blackbird Rusty Blackbird

Common Grackle TOTAL BIRDS COUNT DAY TOTAL BIRDS ONLY IN CP TOTAL SPECIES COUNT DAY TOTAL SPECIES ONLY IN CP

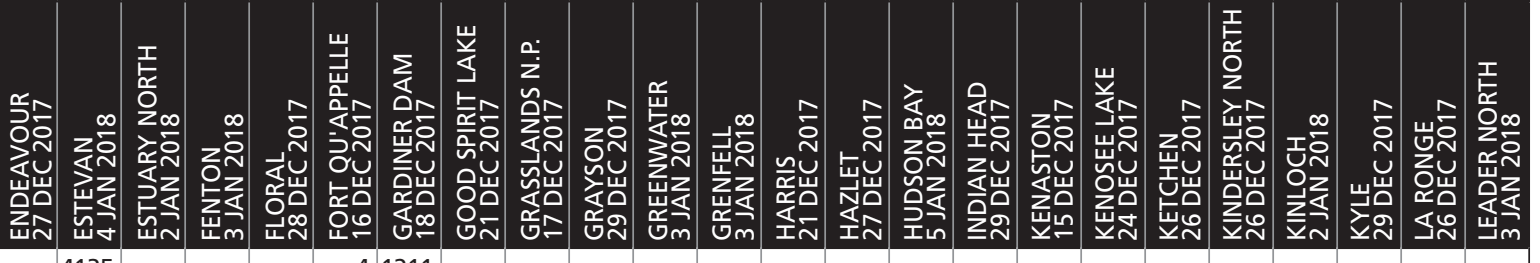
41211

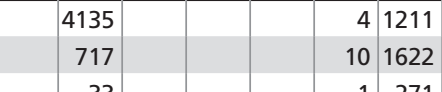

1271

\begin{tabular}{|r|r|r|r|r|r|r|}
\hline & 133 & 116 & & & & \\
\hline 1 & & & & & 1 & \\
\hline 2 & 3 & 63 & & 2 & 10 & 5 \\
\hline & 595 & & 30 & 181 & 125 & 101 \\
\hline & 1 & & 1 & 3 & 3 \\
\hline & & & & $(1)$ & \\
\hline & & & 1 & 2 & 65 \\
\hline & & & & & \\
\hline
\end{tabular}

\begin{tabular}{|r|r|r|r|r|}
\hline$(10)$ & & 11 & 8 & 40 \\
\hline 17 & & & & \\
\hline
\end{tabular}

19

(1)

(10) (26)

\begin{tabular}{|l|l|l|l|l|}
\hline 2 & & & 1 & 6 \\
\hline
\end{tabular}

139

67

90

\begin{tabular}{|l|l|r|}
55 & 22 & 3 \\
\hline
\end{tabular}

\begin{tabular}{|r|r|r|}
\hline 14 & 8 & 2 \\
\hline
\end{tabular}

\begin{tabular}{|l|l|l|}
\hline 27 & 11 & 4 \\
\hline
\end{tabular}

\begin{tabular}{|l|l|l|l|l|}
\hline 9 & 4 & & 9 & 21 \\
\hline
\end{tabular}

2

\begin{tabular}{|r|r|r|r|r|r|r|}
\hline & 1 & 1 & & & \\
\hline & & 3 & & 2 & 1 \\
\hline 1 & 8 & 2 & 1 & 7 & 13 \\
\hline 1 & 4 & 2 & & 5 & 8 \\
\hline & 2 & 1 & & & 1 \\
\hline$(1)$ & & & & & \\
\hline
\end{tabular}

(1)

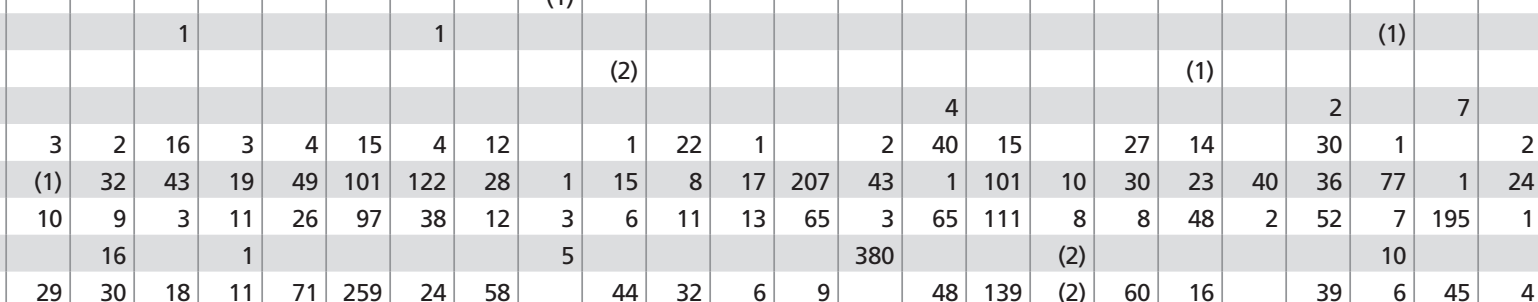

(1)

2
$(1)$

$3 \quad$ (1)

(1)

\begin{tabular}{|l|l|l|l|}
\hline 1 & 4 & 5 & (1)
\end{tabular}

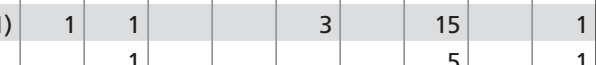

\begin{tabular}{l|l|l|l|l|}
1 & $(1)$ & $(1)$
\end{tabular} \begin{tabular}{|l|l|l|l|l|l|l|l|l|l|l|l|l|l|l|l|l|l|l|l|l|l|l|}
\hline 29 & 30 & 18 & 11 & 71 & 259 & 24 & 58 & 44 & 32 & 6 & 9 & 48 & 139 & $(2)$ & 60 & 16 & 39 & 6 & 45 & 4 \\
\hline
\end{tabular}

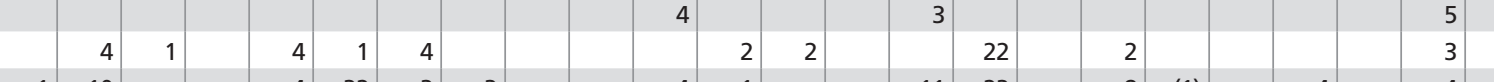
\begin{tabular}{|l|l|l|l|l|l|l|l|l|l|l|l|l|l|l|l|l|}
\hline 1 & 10 & 4 & 32 & 3 & 3 & & 4 & 1 & & 11 & 23 & 8 & $(1)$ & 4 & 4 \\
\hline
\end{tabular}

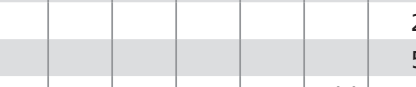

(1)

(1)

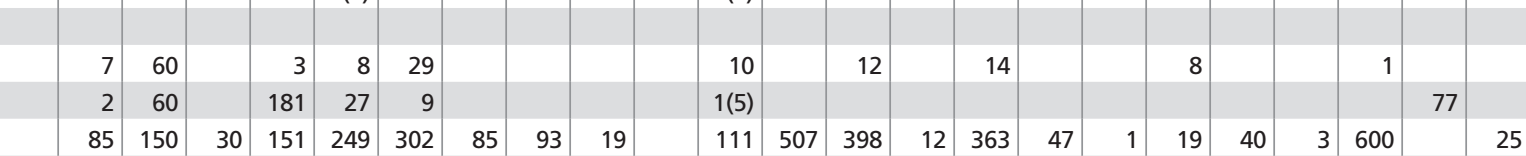
\begin{tabular}{|r|r|r|r|r|r|r|r|r|r|r|r|r|r|r|r|r|r|r|r|r|r|r|}
\hline 10 & 85 & 150 & 30 & 151 & 249 & 302 & 85 & 93 & 19 & & 111 & 507 & 398 & 12 & 363 & 47 & 1 & 19 & 40 & 3 & 600 & 25 \\
\hline
\end{tabular}

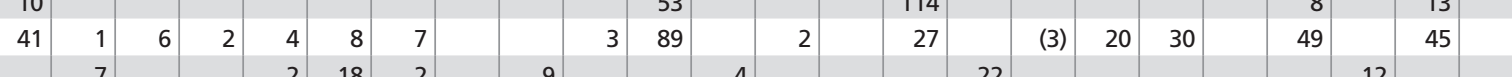

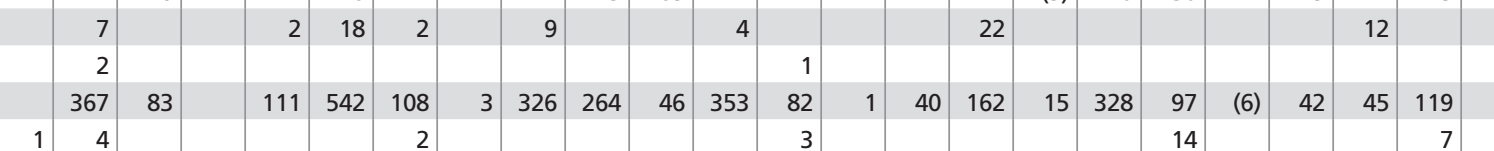
\begin{tabular}{l|l|l|l|l|l|l|l}
\hline & & & \\
& & & $(1)$
\end{tabular}

\begin{tabular}{|l|l|l|l|l|l|}
\hline$(1)$ & & & & & \\
\hline
\end{tabular} \begin{tabular}{|l|l|r|r|r|r|r|r|r|r|r|r|r|r|r|r|r|r|r|r|r|r|r|r|r|}
\hline$(200)$ & 4 & 75 & 1 & & 10 & 52 & 14 & 100 & & & 1016 & 1640 & $(45)$ & 4 & 2000 & 5 & 19 & 6 & 29 & 500 & 3 \\
\hline
\end{tabular} \begin{tabular}{|l|l|l|l|r|r|r|r|r|r|r|r|r|r|r|r|r|r|r|r|r|r|r|}
\hline 7 & 6 & 1 & 3 & 5 & & 1 & 2 & 1 & 2 & & 6 & & $(1)$ & 3 & 15 & \\
\hline
\end{tabular}

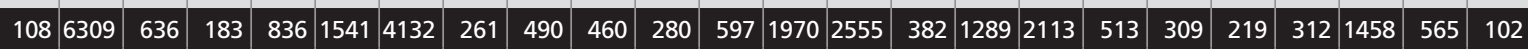

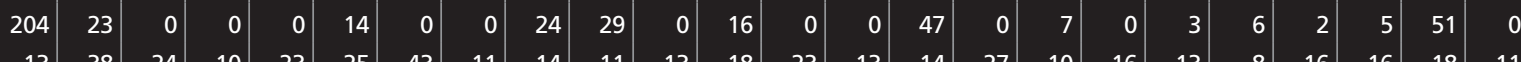
\begin{tabular}{r|r|r|r|r|r|r|r|r|r|r|r|r|r|r|r|r|r|r|r|r|r|r|r|}
13 & 38 & 24 & 10 & 23 & 25 & 43 & 11 & 14 & 11 & 13 & 18 & 23 & 13 & 14 & 27 & 10 & 16 & 13 & 8 & 16 & 16 & 18 & 11 \\
5 & 3 & 0 & 0 & 0 & 6 & 0 & 0 & 6 & 3 & 0 & 2 & 0 & 0 & 3 & 0 & 3 & 0 & 3 & 1 & 2 & 5 & 2 & 0
\end{tabular} 
TABLE 3. Species found on 6 or more counts; ( ) = seen during count period (CP).

\begin{tabular}{|c|c|c|c|c|c|c|c|c|c|c|c|c|c|c|c|c|c|c|c|c|c|c|c|c|}
\hline SPECIES & 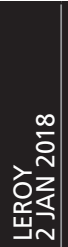 & 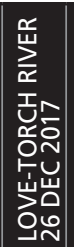 & 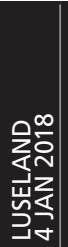 & 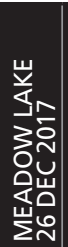 & 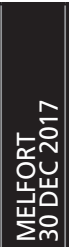 & 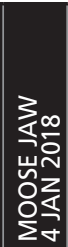 & 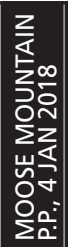 & 周 & 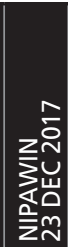 & 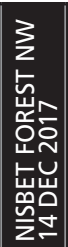 & 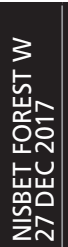 & 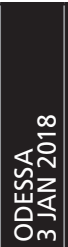 & 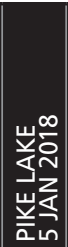 & 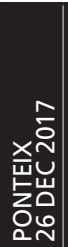 & 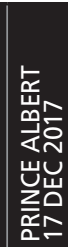 & 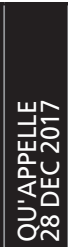 & 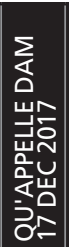 & Ẽ & 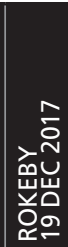 & 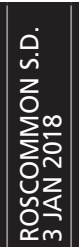 & 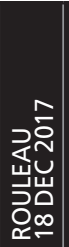 & 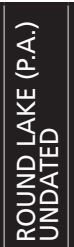 & 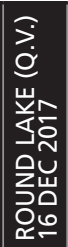 & 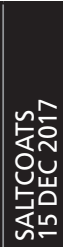 \\
\hline Canada Goose & & & & & & & & 51 & & & & & & & & & 625 & 186 & & & & & & \\
\hline Mallard & & & & & & 5 & & & & & & & & & & & 187 & 15 & & & & & & \\
\hline Common Goldeneye & & & & & & & & & 3 & & & & & & (6) & & 155 & (1) & & & & & & \\
\hline Gray Partridge & 5 & & 79 & & 2 & & & 130 & & & & 10 & & 18 & & (8) & & 87 & 52 & 13 & & & & 28 \\
\hline Ring-necked Pheasant & & & & & & 2 & & (1) & & & & & & (1) & & & & & & & & & & \\
\hline Ruffed Grouse & & 5 & & & & & 2 & & & & (1) & 4 & 4 & & 3 & 2 & & & 4 & 4 & & & & 5 \\
\hline Sharp-tailed Grouse & 6 & & & & & & & 25 & & & (1) & 24 & 87 & 18 & 1 & 7 & 2 & (1) & 57 & & & & & 4 \\
\hline Rock Pigeon & 41 & 97 & 33 & & 119 & 334 & 3 & 73 & 163 & & 10 & 8 & 137 & 12 & 335 & 126 & 294 & 736 & 251 & 17 & 91 & & 12 & \\
\hline Eurasian Collared-Dove & & & 12 & & 7 & 65 & & 47 & & & & & & 104 & & 2 & 7 & & & & (1) & & & 14 \\
\hline Mourning Dove & & & & & & & & & 2 & & & & & & & & & & & & & & & \\
\hline Bald Eagle & & (1) & & 1 & & & & & & & & 2 & & & & & 19 & & 2 & & & (1) & 3 & \\
\hline Sharp-shinned Hawk & & (1) & & & & & & & & & & & & & & & & 2 & & & & & & \\
\hline Northern Goshawk & & 1 & & & 1 & & & & & & & & 1 & & & & & 1 & & 1 & & & & \\
\hline Rough-legged Hawk & & & & & & & & & & & & & & & & & & (1) & & & & & & \\
\hline Golden Eagle & & & & & & & & & & & & & & & & & 1 & & & & & & 1 & \\
\hline Great Horned Owl & & & 5 & & (1) & 3 & & 25 & & & (1) & 4 & 3 & 6 & (1) & 2 & & 5 & 1 & (1) & 3 & & & \\
\hline Snowy Owl & & & 7 & & (1) & 3 & & 6 & & & & 2 & 2 & 6 & & (1) & & 45 & 3 & & 44 & & & 1 \\
\hline Downy Woodpecker & 3 & 13 & 2 & & 5 & 7 & 12 & (2) & 8 & 3 & 2 & & 25 & 2 & 18 & 17 & 5 & 17 & 19 & 8 & 1 & 1 & 2 & 24 \\
\hline Hairy Woodpecker & 1 & 11 & (1) & & 4 & 2 & 3 & & 6 & 2 & 1 & 6 & 11 & & 18 & 18 & 3 & 3 & 16 & 12 & & 1 & 3 & 21 \\
\hline Black-backed Woodpecker & & 4 & & & & & & & & & & & & & & & & & & & & & & \\
\hline Northern Flicker & & & 1 & & & & & & & & & 4 & & & (1) & & 1 & (1) & 2 & & & & & \\
\hline Pileated Woodpecker & & (1) & & & (1) & & 1 & & (1) & & & 2 & (1) & & 3 & & & & & & & & 2 & 1 \\
\hline Merlin & & & & & & 1 & & & & & & & 1 & & (1) & & & 1 & 1 & & & & & \\
\hline Prairie Falcon & & & & & & & & & & & & & & & & & & & & & & & & \\
\hline Northern Shrike & & (1) & & & & & & & & & 1 & & 2 & 1 & 1 & & & 1 & 1 & & & & (1) & \\
\hline Gray Jay & & 12 & & & & & & & & & & & & & 3 & & & & & & & & & \\
\hline Blue Jay & & 29 & 13 & 10 & 5 & 3 & 8 & 2 & 24 & 2 & 4 & 2 & 13 & & 42 & & 5 & 7 & 9 & 16 & & & 8 & 9 \\
\hline Black-billed Magpie & 37 & 69 & 125 & 16 & 48 & 78 & 14 & 68 & 36 & 2 & 9 & 32 & 225 & 29 & 205 & 129 & 85 & 68 & 60 & 66 & 3 & 1 & 16 & 10 \\
\hline Common Raven & 48 & 101 & 7 & 52 & 354 & 31 & 8 & 2 & 219 & & 23 & 25 & 114 & & 506 & 153 & 37 & 53 & 53 & 42 & 5 & 1 & 32 & 23 \\
\hline Horned Lark & & & 6 & & & 42 & & 595 & & & & & & 674 & & 19 & & & & & & & 1 & \\
\hline Black-capped Chickadee & 26 & 143 & 16 & 2 & 42 & 20 & 30 & & 68 & 20 & 27 & 20 & 428 & & 246 & 107 & 34 & 153 & 126 & 106 & & 14 & 51 & 105 \\
\hline Boreal Chickadee & & 4 & & & & & & & & & & & & & 2 & & & & & & & 2 & & \\
\hline Red-breasted Nuthatch & & 19 & 2 & & 9 & 10 & 1 & (1) & 13 & & & & 2 & & 5 & 4 & 5 & 45 & 2 & & & & & 6 \\
\hline White-breasted Nuthatch & 2 & 8 & & & 4 & 5 & 2 & & 7 & 2 & (1) & & 16 & & 19 & 7 & 6 & 11 & 4 & 7 & & & 12 & 19 \\
\hline Brown Creeper & & & & & (1) & & & 1 & & & & & & & & & & 2 & & & & & & \\
\hline Golden-crowned Kinglet & & & & & & & & (3) & & & & & & & 1 & & 3 & 1 & & & & & & \\
\hline American Robin & & (1) & (1) & & & 3 & & & & & & 2 & & & 1 & & 2 & 1 & & & & & & \\
\hline Varied Thrush & & & & & & & & & & & & & & & & & 1 & 1 & & & & & & \\
\hline European Starling & 5 & 10 & 2 & & 60 & 67 & & 1 & 90 & & & 6 & 22 & 5 & 41 & 16 & 8 & 12 & & & & & & \\
\hline Bohemian Waxwing & 9 & (1) & & & 1262 & 55 & & (2) & 115 & & & & 12 & 75 & 4424 & & & 30 & 1 & & & & & \\
\hline House Sparrow & 198 & 62 & 14 & & 400 & 429 & 14 & 1081 & 32 & & 10 & 40 & 327 & 449 & 548 & 251 & 360 & 2093 & 489 & 81 & 87 & & 107 & 189 \\
\hline Evening Grosbeak & & 177 & & & & & & & 33 & & 70 & & & & 42 & & & & & & & 10 & & \\
\hline Pine Grosbeak & & 190 & 2 & 28 & 3 & & 14 & & 104 & 12 & 29 & 2 & 94 & & 298 & 3 & 21 & 1 & & 58 & & 6 & & 15 \\
\hline House Finch & & & (1) & & & 103 & & 1 & & & & & 27 & 1 & 2 & 2 & 15 & 195 & & & & & & \\
\hline Purple Finch & & & & & & & & & & & & & & & (1) & 5 & & & & & 2 & & & \\
\hline Common Redpoll & 72 & 426 & 340 & & 57 & 6 & 550 & 203 & 148 & 10 & 381 & & 294 & 6 & 817 & 663 & 26 & 15 & 401 & 820 & & 10 & 50 & 167 \\
\hline Hoary Redpoll & & 3 & & & 2 & & & 2 & 5 & & & & 12 & & (1) & & & & 5 & 2 & & & 1 & \\
\hline White-winged Crossbill & & & & & & & & & 5 & & & & & & & & & & & 6 & & & & \\
\hline Pine Siskin & 5 & & & & 9 & & & & & & & & 4 & & & & & & & 2 & & & & 7 \\
\hline American Goldfinch & & & & & & 2 & & & & & & & & & (6) & & & & & (1) & & & & \\
\hline Snow Bunting & 170 & 940 & 90 & 23 & (9) & 125 & & 3531 & & & & 10 & 700 & 350 & 20 & 256 & & 151 & 60 & 425 & 18 & & & 203 \\
\hline White-throated Sparrow & & & & & & & & & & & & & & & & 10 & & 1 & & & & & & \\
\hline Dark-eyed Junco & & 5 & (1) & & & 14 & & & 5 & & & & & & 1 & 1 & & 27 & & 1 & & & & 10 \\
\hline Red-winged Blackbird & & & & & & 5 & & & & & & & & & 1 & & & & & & & & & \\
\hline Rusty Blackbird & & & & & & & & & & & & 4 & 3 & & & & & & & & & & & \\
\hline Common Grackle & & & & & & 2 & & & & & & & & & & & & 1 & & & & & & \\
\hline TOTAL BIRDS COUNT DAY & 728 & 2332 & 756 & 132 & 2404 & 1424 & 662 & 5846 & 1093 & 53 & 567 & 209 & 2566 & 1761 & 7605 & 1810 & 1911 & 3971 & 1620 & 1687 & 254 & 47 & 303 & 862 \\
\hline TOTAL BIRDS ONLY IN CP & 0 & 6 & 4 & 0 & 13 & 0 & 0 & 10 & 1 & 0 & 4 & 0 & 1 & 1 & 19 & 9 & 0 & 4 & 0 & 2 & 1 & 1 & 1 & 0 \\
\hline TOTAL SPECIES COUNT DAY & 16 & 23 & 18 & 7 & 20 & 28 & 14 & 19 & 21 & 8 & 12 & 20 & 26 & 19 & 28 & 23 & 29 & 33 & 24 & 19 & 9 & 10 & 16 & 21 \\
\hline TOTAL SPECIES ONLY IN CP & 0 & 6 & 4 & 0 & 5 & 0 & 0 & 6 & 1 & 0 & 4 & 0 & 1 & 1 & 9 & 2 & 0 & 4 & 0 & 2 & 1 & 1 & 1 & 0 \\
\hline
\end{tabular}




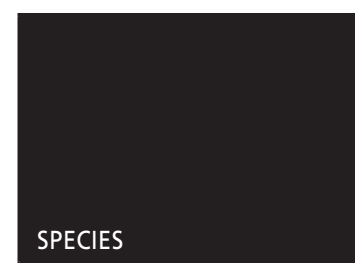

Canada Goose
Mallard

Common Goldeneye Gray Partridge

Ring-necked Pheasant Ruffed Grouse

Sharp-tailed Grouse Rock Pigeon

Eurasian Collared-Dove Mourning Dove Bald Eagle

Sharp-shinned Hawk

Northern Goshawk

Rough-legged Hawk Golden Eagle

Great Horned Owl Snowy Owl

Downy Woodpecker

Hairy Woodpecker Black-backed Woodpecker Northern Flicker

Pileated Woodpecker Prairie Falcon Northern Shrike Gray Jay Blue Jay

Black-billed Magpie

Common Raven

Horned Lark

Black-capped Chickadee

Boreal Chickadee

Red-breasted Nuthatch

White-breasted Nuthatch

Brown Creeper

Golden-crowned Kinglet American Robin Varied Thrush

European Starling

Bohemian Waxwing

House Sparrow

Evening Grosbeak

Pine Grosbeak

House Finch

Purple Finch

Common Redpoll Hoary Redpoll

White-winged Crossbill Pine Siskin

American Goldfinch Snow Bunting

White-throated Sparrow

Dark-eyed Junco

Red-winged Blackbird Rusty Blackbird

Common Grackle

TOTAL BIRDS COUNT DAY

TOTAL BIRDS ONLY IN CP

TOTAL SPECIES COUNT DAY

TOTAL SPECIES ONLY IN CP

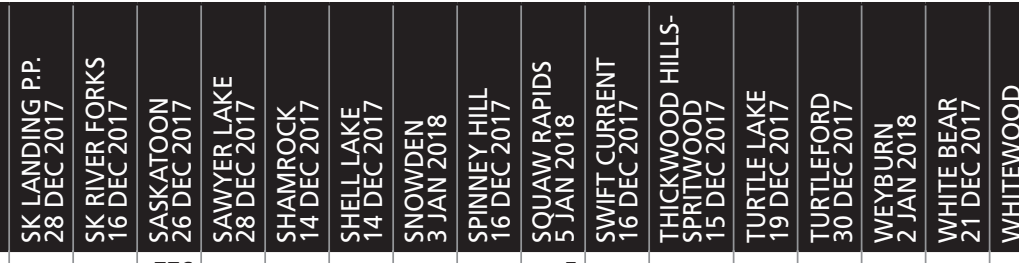

\begin{tabular}{|c|c|c|c|c|c|c|c|c|}
\hline & & 776 & & & & & & 5 \\
\hline 1 & & 9 & & & & & & \\
\hline & 4 & 121 & & & & & & 138 \\
\hline 16 & & 9 & & 36 & & 4 & & \\
\hline 4 & & & & 17 & & & & \\
\hline & & 1 & 2 & & & 3 & & \\
\hline 36 & & 35 & & 17 & & 9 & & \\
\hline 17 & & 1314 & 48 & 35 & 10 & & 23 & \\
\hline
\end{tabular}

\section{5}

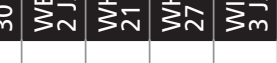

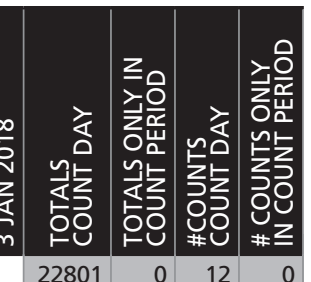

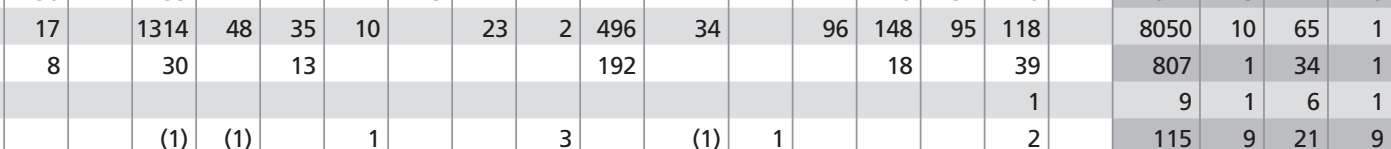

\begin{tabular}{|r|r|r|r|r|r|r|r|r|r|}
\hline & & (1) & (1) & & 1 & & & 3 & \\
\hline 1 & & & $(1)$ & & & & 1 & & \\
\hline 2 & & 1 & & 1 & & & & & \\
\hline 2 & & 1 & & 9 & & & & & \\
\hline 2 & & & & 9 & & & & & \\
\hline 4 & 2 & 51 & & 2 & 3 & 8 & 1 & 2 & 14 \\
\hline
\end{tabular}

\begin{tabular}{|l|l|l|}
\hline 10 & 1 & \\
\hline & & \\
\hline
\end{tabular}

\begin{tabular}{|l|l|l|l|}
8283 & 0 & 14 & 0 \\
\hline 759
\end{tabular}

\begin{tabular}{|l|r|r|r|r|r|r|r|}
\hline 28 & 34 & 18 & 59 & 1050 & 38 & 33 & 5 \\
\hline
\end{tabular}

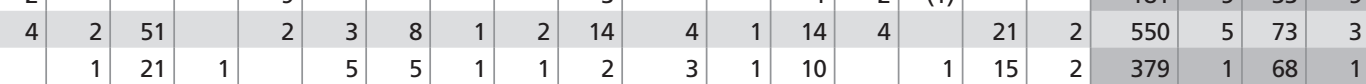

10

12

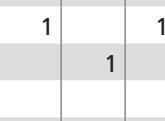

\section{(1)}

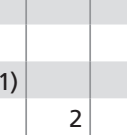

(1)

$8 \quad 2$

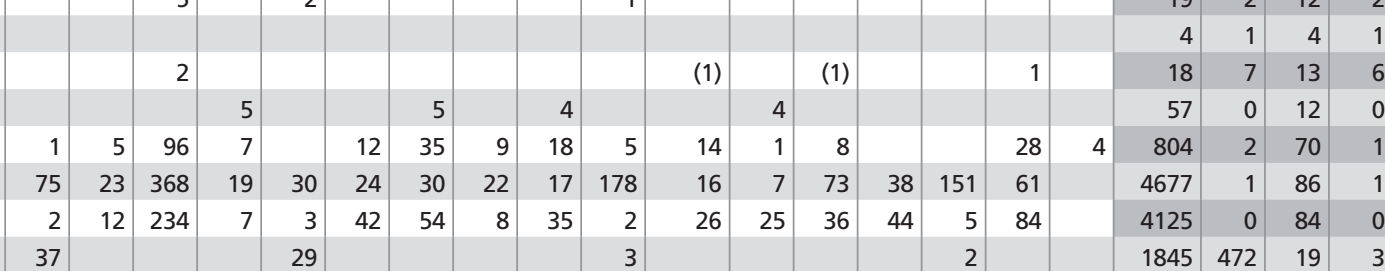

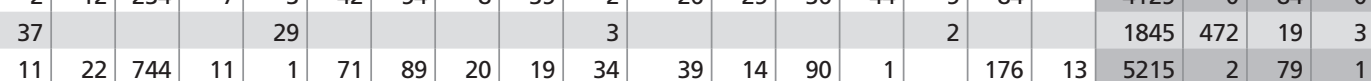

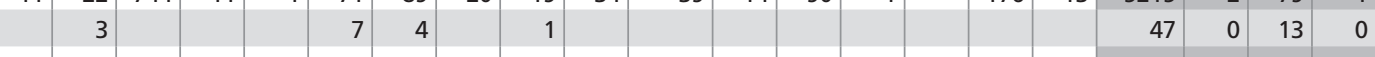

\begin{tabular}{|l|l|l|r|r|r|r|r|r|r|r|r|r|r|r|r|r|r|}
\hline 96 & 1 & 4 & 2 & 50 & 1 & 1 & 4 & 8 & 379 & 4 & 44 & 3 \\
\hline
\end{tabular}

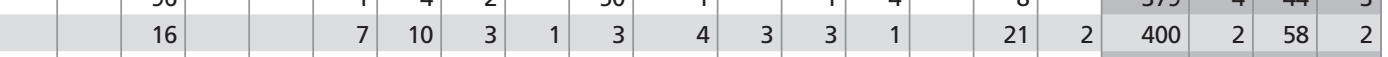

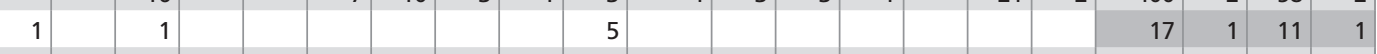
\begin{tabular}{|l|r|r|r|r|r|r|r|r|r|}
\hline 5 & 3 & 3 & & 31 & 3 & 10 & 1 \\
\hline
\end{tabular}

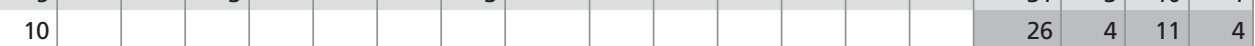

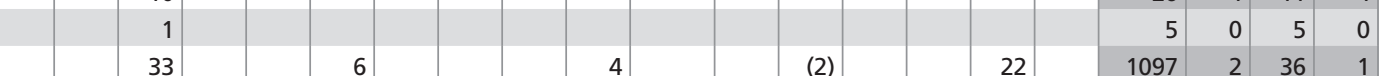

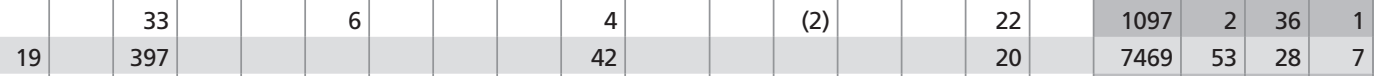
\begin{tabular}{|r|r|r|r|r|r|r|r|r|r|r|r|r|r|r|r|r|r|r|r|r|r|r|}
\hline 400 & 5 & 2668 & 88 & 1273 & 2 & 63 & 10 & 25 & 1367 & 6 & & 141 & 220 & 946 & 340 & 100 & 22475 & 0 & 78 & 0 \\
\hline
\end{tabular}

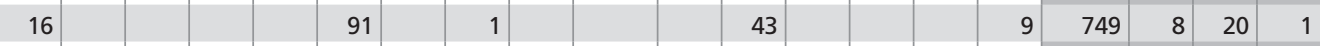
\begin{tabular}{|r|r|r|r|r|r|r|r|r|r|r|r|r|r|r|r|r|r|r|r|r|}
\hline 48 & 59 & 7 & 68 & 157 & 5 & 6 & 6 & 15 & 19 & 64 & & 21 & 8 & 2153 & 11 & 62 & 2 \\
\hline
\end{tabular}

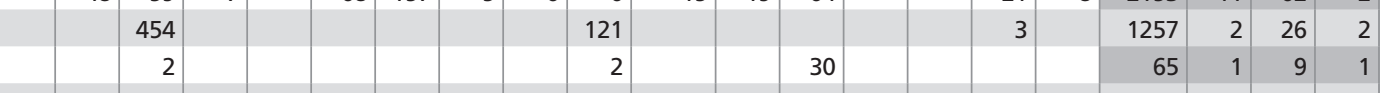
\begin{tabular}{|l|r|r|r|r|r|r|r|r|r|r|r|r|r|r|r|r|r|r|r|r|r|}
\hline 280 & 186 & 251 & 16 & 45 & 19 & 355 & 22 & & $(1)$ & 56 & 12 & 284 & 34 & 105 & 894 & 6 & 14307 & 9 & 78 & 3 \\
\hline
\end{tabular}

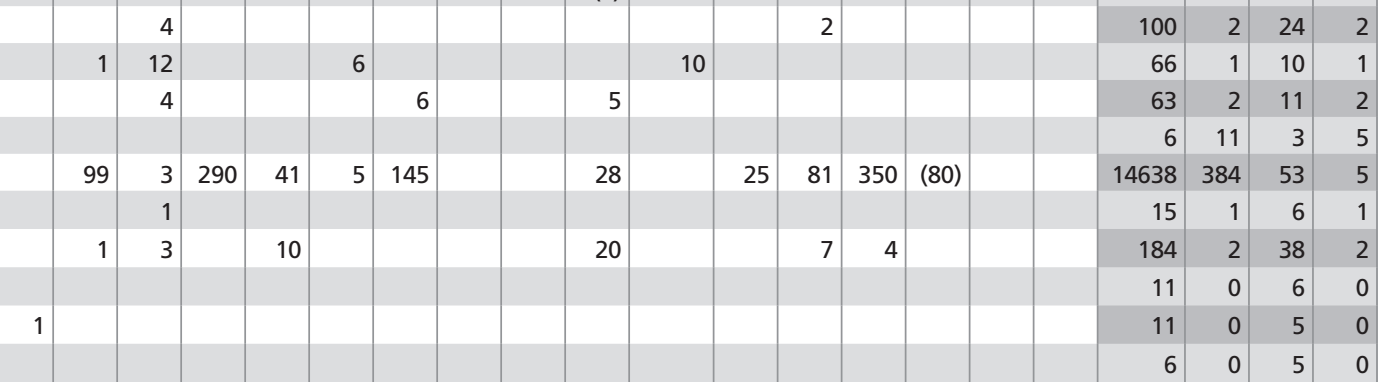

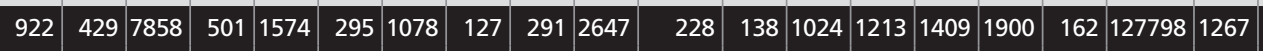

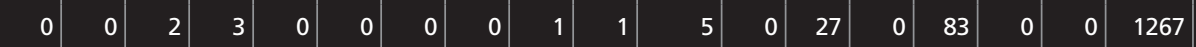
\begin{tabular}{|l|r|r|r|r|r|r|r|r|r|r|r|r|r|r|r|r|r|}
23 & 16 & 42 & 12 & 19 & 21 & 20 & 13 & 20 & 32 & 13 & 16 & 22 & 17 & 10 & 24 & 11 & 92 \\
\hline
\end{tabular}

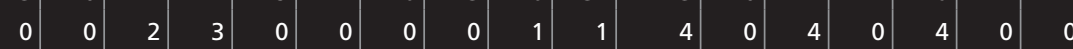


TABLE 4. Species found in fewer than 6 counts.

\begin{tabular}{|c|c|}
\hline SPECIES & LOCALITY AND NUMBER ( ${ }^{*}=$ SEEN DURING COUNT PERIOD) \\
\hline Snow Goose & Estevan $(21 *)$ \\
\hline Cackling Goose & Coronach (56), Estevan (37), Gardiner Dam (17) \\
\hline Gadwall & Gardiner Dam (1), Qu'Appelle Dam (1) \\
\hline Northern Pintail & Gardiner Dam (1) \\
\hline Green-winged Teal & Gardiner Dam (1), Saskatchewan Landing (1) \\
\hline Redhead & Gardiner Dam (13) \\
\hline Ring-necked Duck & Crooked Lake (1) \\
\hline Greater Scaup & Estevan (1), Gardiner Dam (38) \\
\hline Lesser Scaup & Crooked Lake (3), Estevan (3), Gardiner Dam (4) \\
\hline Bufflehead & Estevan (3), Gardiner Dam (11) \\
\hline Hooded Merganser & Estevan (2) \\
\hline Common Merganser & Coronach (4), Gardiner Dam (57), Squaw Rapids (6) \\
\hline Ruddy Duck & Estevan (1), Qu'Appelle Dam (1) \\
\hline Spruce Grouse & Creighton (1), Love-Torch River (3) \\
\hline Willow Ptarmigan & Creighton (16), La Ronge (50*) \\
\hline American Coot & Estevan (2) \\
\hline Wilson's Snipe & Qu'Appelle Dam (1) \\
\hline Herring Gull & Gardiner Dam (2) \\
\hline Glaucous Gull & Gardiner Dam (1) \\
\hline Red-tailed Hawk & Coronach (1), Round Lake (Qu'Appelle Valley) (2) \\
\hline Northern Hawk Owl & Endeavour $(1 *)$ \\
\hline Great Gray Owl & Endeavour (1), Kinloch (1), La Ronge (1*), Prince Albert (1*) \\
\hline Long-eared Owl & White Bear $(1 *)$ \\
\hline Short-eared Owl & Kyle (1*), Moose Jaw (2), Ponteix (1), White Bear (1*) \\
\hline Northern Saw-whet Owl & Dundurn (1), Endeavour (1), Kyle (1*), Qu'Appelle Dam (1) \\
\hline Red-bellied Woodpecker & Estuary North (1), Saskatoon (1*) \\
\hline American Three-toed Woodpecker & Creighton (1), Prince Albert (2), Saltcoats (1), Shell Lake (1) \\
\hline Gyrfalcon & Clark's Crossing (1), Kyle (1*) \\
\hline American Crow & Regina (1), Round Lake (1), Saskatoon (3), Turtle Lake (3) \\
\hline Townsend's Solitaire & Swift Current (1) \\
\hline Hermit Thrush & Swift Current (1) \\
\hline Cedar Waxwing & Biggar (88), Turtle Lake (20), Turtleford (23*) \\
\hline Red Crossbill & Broadview $\left(2^{*}\right)$, Melfort (11), Nipawin (7) \\
\hline Lapland Longspur & Leroy (100), Ponteix (3), Qu'Appelle (10), \\
\hline American Tree Sparrow & Craven (1), Eastend (19) \\
\hline Chipping Sparrow & Prince Albert $(1 *)$ \\
\hline Harris's Sparrow & Craven (1), Grasslands N.P. (1*), Morse (2) \\
\hline Yellow-headed Blackbird & Indian Head (2) \\
\hline Western Meadowlark & Kinloch $(1 *)$ \\
\hline Brown-headed Cowbird & Avonlea (1) \\
\hline Brewer's Blackbird & Rokeby (1), Whitewood (1), Wingard (1) \\
\hline
\end{tabular}

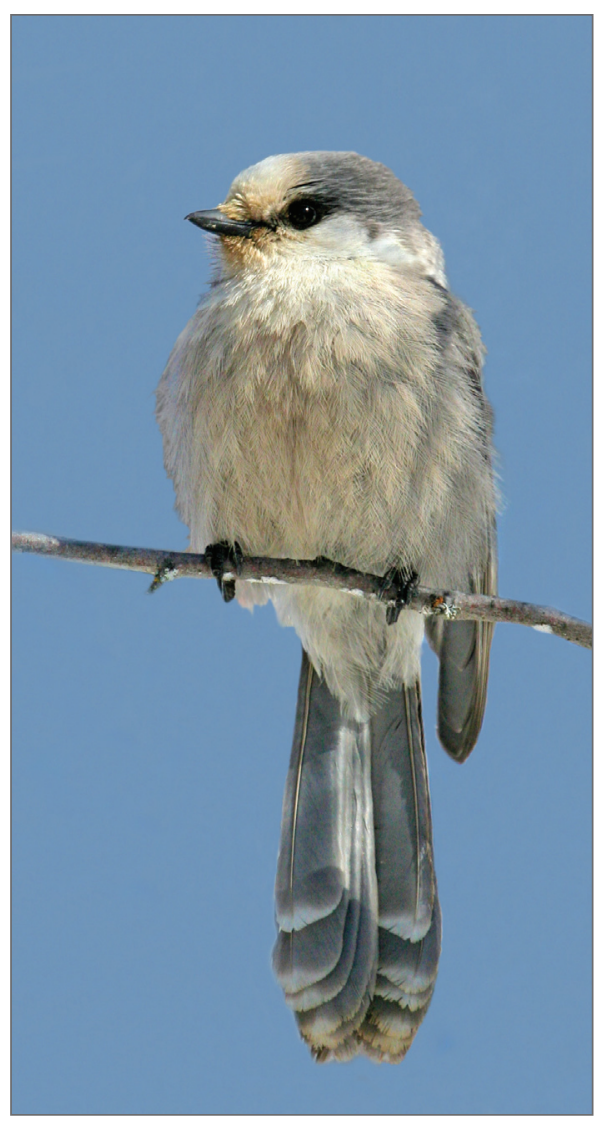

Gray Jay. Photo credit: Nick Saunders

\section{TABLE 5. Birds not identified to species.}

\begin{tabular}{|r|r|}
\hline CATEGORY & $\begin{array}{r}\text { LOCALITY AND NUMBER } \\
\text { (*=SEEN DURING } \\
\text { COUNT PERIOD) }\end{array}$ \\
\hline Small Accipiter & Regina (2) \\
Accipiter sp. & $\begin{array}{r}\text { Biggar (1) } \\
\text { Churchbridge (1) } \\
\text { Craven (1) }\end{array}$ \\
Woodpecker sp. & Squaw rapids (1*) \\
\hline Falcon sp. & Pike Lake (2) \\
Large Falcon sp. & Morse (1*) \\
Crossbill sp. & Ponteix (1) \\
\hline Blackbird sp. & Chatsworth (1*) \\
& Grenfell (1) \\
& Regina (1) \\
\hline
\end{tabular}

TABLE 6. New (in bold and italics) and tying high counts for individual species 2017.

\begin{tabular}{|c|c|c|c|c|}
\hline LOCATION & 2017 COUNT & SPECIES & $\begin{array}{r}\text { PREVIOUS } \\
\text { HIGH }\end{array}$ & LOCATION, YEAR \\
\hline Estevan & 21 & $\begin{array}{l}\text { Snow Goose } \\
\text { (count week) }\end{array}$ & 4 & Weyburn 1999, Regina 2015, 2016 \\
\hline Gardiner Dam & 38 & Greater Scaup & 23 & Gardiner Dam 2016 \\
\hline Swift Current & 196 & Eurasian Collared-Dove & 116 & Swift Current 2009 \\
\hline Regina & 45 & Snowy Owl & 45 & Regina 2011 \\
\hline Estuary North, Saskatoon & 1 & Red-bellied Woodpecker & 1 & $\begin{array}{l}\text { Regina 2001, 2016; Yorkton 2007, } \\
\text { Crooked River 2010, Grenfell 2013, Nipawin } 2016\end{array}$ \\
\hline Ft. Qu'Appelle & 32 & White-breasted Nuthatch & 32 & Ft. Qu'Appelle 2013 \\
\hline Swift Current & 1 & Hermit Thrush & 1 & MacDowall 1997, Estevan 2003, Regina 2006 \\
\hline Qu'Appelle & 10 & White-throated Sparrow & 10 & Armit 2005 \\
\hline Indian Head & 2 & Yellow-headed Blackbird & 2 & Indian Head 2001 \\
\hline
\end{tabular}

